\title{
Worldsheet theory of light-cone gauge noncritical strings on higher genus Riemann surfaces
}

\author{
Nobuyuki Ishibashi ${ }^{a}$ and Koichi Murakami ${ }^{b}$ \\ ${ }^{a}$ Graduate School of Pure and Applied Sciences, University of Tsukuba, \\ Tsukuba, Ibaraki 305-8571, Japan \\ ${ }^{b}$ National Institute of Technology, Kushiro College, \\ Otanoshike-Nishi 2-32-1, Kushiro, Hokkaido 084-0916, Japan \\ E-mail: ishibash@het.ph.tsukuba.ac.jp, koichi@kushiro-ct.ac.jp
}

ABSTRACT: It is possible to formulate light-cone gauge string field theory in noncritical dimensions. Such a theory corresponds to conformal gauge worldsheet theory with nonstandard longitudinal part. We study the longitudinal part of the worldsheet theory on higher genus Riemann surfaces. The results in this paper shall be used to study the dimensional regularization of light-cone gauge string field theory.

Keywords: String Field Theory, BRST Quantization, Conformal Field Models in String Theory, Superstrings and Heterotic Strings

ARXiv EPRINT: 1603.08337 


\section{Contents}

1 Introduction $\quad 1$

2 Bosonic $X^{ \pm}$CFT 2

2.1 Correlation functions on higher genus Riemann surfaces 4

3 Supersymmetric $X^{ \pm}$CFT 10

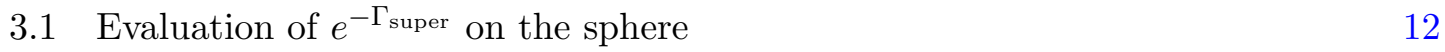

$\begin{array}{ll}3.2 e^{-\Gamma_{\text {super }}} \text { for higher genus Riemann surfaces } & 18\end{array}$

3.3 Correlation functions of supersymmetric $X^{ \pm}$CFT 20

4 Discussions $\quad 22$

A Definition of the path integral of $X^{ \pm}$variables $\quad 22$

B Arakelov metric and Arakelov Green's function 24

C Calculations of correlation functions of bosonic $X^{ \pm}$CFT $\quad 25$

$\begin{array}{lll}\text { C.1 Evaluation of }\left\langle\partial X^{-}(z)\right\rangle_{\rho} & 25\end{array}$

$\begin{array}{lll}\text { C.2 Energy-momentum tensor } & 27\end{array}$

$\begin{array}{ll}\text { D Interaction points and the odd moduli } & 30\end{array}$

E Calculations of correlation functions of supersymmetric $X^{ \pm}$CFT $\quad 31$

E.1 An expression in terms of the superfield $\rho(\mathbf{z}) \quad 33$

E.2 Energy-momentum tensor 34

\section{Introduction}

Since light-cone gauge string field theory is a completely gauge fixed theory, there is no problem in formulating it in noncritical dimensions. It should be possible to find the worldsheet theory in the conformal gauge describing such a string theory, in which the spacetime Lorentz invariance shall be broken. In [1,2], we have constructed the longitudinal part of the worldsheet theory which we call the $X^{ \pm}$CFT. The $X^{ \pm}$CFT turns out to be a conformal field theory with the right central charge so that the whole worldsheet theory is BRST invariant. The light-cone gauge superstring field theory in noncritical dimensions can be used [3-6] to regularize the so-called contact term divergences [7-11], in the case of tree level amplitudes. The supersymmetric $X^{ \pm}$CFT plays crucial roles in studying such a regularization. 


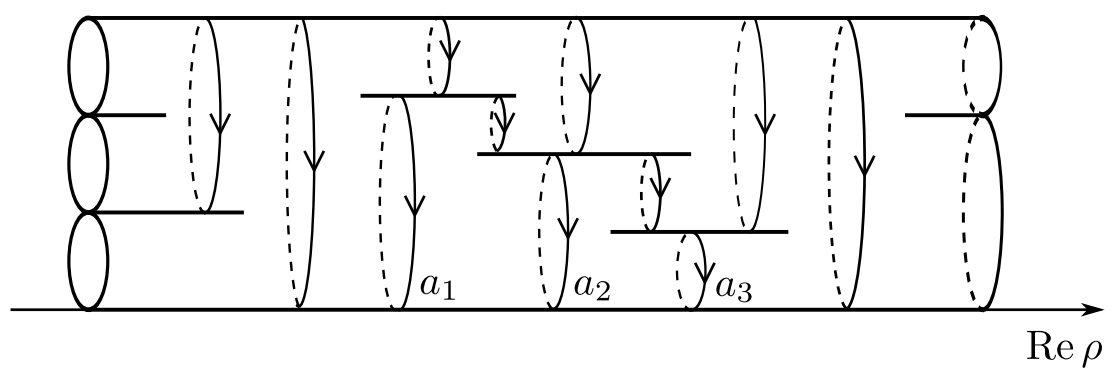

Figure 1. A string diagram with 3 incoming, 2 outgoing strings and 3 loops.

In this paper, we would like to study the $X^{ \pm}$CFT on higher genus Riemann surfaces. In a previous paper [12], we have dealt with the bosonic $X^{ \pm}$CFT on higher genus Riemann surfaces, but we have not investigated its properties in detail. In this paper, we will define and calculate the correlation functions of bosonic and supersymmetric $X^{ \pm} \mathrm{CFT}$ on higher genus Riemann surfaces and explore various properties of the theory. The results in this paper will be used in a forthcoming publication, in which we discuss the dimensional regularization of the multiloop amplitudes of light-cone gauge superstring field theory.

The organization of this paper is as follows. In section 2, the bosonic $X^{ \pm}$CFT is studied. We calculate the correlation functions based on the results in [12]. In section 3, we deal with the supersymmetric $X^{ \pm}$CFT. In [2], we have given a way to calculate the correlation functions of the supersymmetric $X^{ \pm}$CFT on a surface of genus 0 , but it is a bit unwieldy. In this paper, we develop an alternative method to calculate them, apply it to higher genus case and explore various properties of the supersymmetric $X^{ \pm}$CFT. Section 4 is devoted to discussions. In the appendices, we give details of definitions and calculations which are not included in the text.

\section{Bosonic $X^{ \pm}$CFT}

It is straightforward to calculate the amplitudes of light-cone gauge bosonic string field theory perturbatively by using the old-fashioned perturbation theory and Wick rotation. Each term in the expansion corresponds to a light-cone gauge Feynman diagram for strings. A typical diagram is depicted in figure 1 . A Wick rotated $g$-loop $N$-string diagram is conformally equivalent to an $N$ punctured genus $g$ Riemann surface $\Sigma$. The amplitudes are given by an integral of correlation functions of vertex operators on $\Sigma$ over the moduli parameters.

As has been shown in $[1,12]$, the amplitudes in $d \neq 26$ dimensions can be cast into the conformal gauge expression using the worldsheet theory with the field contents

$$
X^{+}, \quad X^{-}, \quad X^{i}, \quad b, \quad c, \quad \bar{b}, \quad \bar{c},
$$

in which the reparametrization ghosts $b, c, \bar{b}, \bar{c}$ and the longitudinal variables $X^{ \pm}$are added to the original light-cone variables $X^{i}(i=1, \ldots, d-2)$. The worldsheet action for the longitudinal variables is given by

$$
S\left[\hat{g}_{z \bar{z}}, X^{ \pm}\right]=-\frac{1}{4 \pi} \int d z \wedge d \bar{z} i\left(\partial X^{+} \bar{\partial} X^{-}+\partial X^{-} \bar{\partial} X^{+}\right)+\frac{d-26}{24} \Gamma\left[\hat{g}_{z \bar{z}}, X^{+}\right] .
$$


Here the metric on the worldsheet is taken to be $d s^{2}=2 \hat{g}_{z \bar{z}} d z d \bar{z}$ and $\Gamma$ is the Liouville action

$$
\Gamma\left[\hat{g}_{z \bar{z}}, X^{+}\right]=-\frac{1}{2 \pi} \int d z \wedge d \bar{z} i\left(\partial \phi \bar{\partial} \phi+\hat{g}_{z \bar{z}} \hat{R} \phi\right),
$$

where the Liouville field $\phi$ is given by

$$
\phi \equiv \ln \left(-4 \partial X^{+} \bar{\partial} X^{+}\right)-\ln \left(2 \hat{g}_{z \bar{z}}\right)
$$

and $\hat{R}$ is the scalar curvature derived from the metric $\hat{g}$. The theory with the action $(2.2)$ turns out to be a conformal field theory which we call the $X^{ \pm}$CFT.

In order for the action to be well-defined, $d s^{2}=-4 \partial X^{+} \bar{\partial} X^{+} d z d \bar{z}$ should be a welldefined metric on the worldsheet at least at generic points. Hence we should always consider the theory in the presence of the vertex operator insertions

$$
\prod_{r=1}^{N} e^{-i p_{r}^{+} X^{-}}\left(Z_{r}, \bar{Z}_{r}\right)
$$

with $p_{r}^{+} \neq 0(r=1, \cdots, N)$ and $N \geq 3$. The amplitudes with such insertions correspond to light-cone diagrams with external lines at $z=Z_{r}$ which have string length $\alpha_{r}=2 p_{r}^{+}$. With the insertion of these vertex operators, $X^{+}$possesses a classical background

$$
X_{\mathrm{cl}}^{+}(z, \bar{z})=-\frac{i}{2}(\rho(z)+\bar{\rho}(\bar{z}))
$$

where $\rho(z)$ is given by

$$
\rho(z)=\sum_{r=1}^{N} \alpha_{r}\left[\ln E\left(z, Z_{r}\right)-2 \pi i \int_{P_{0}}^{z} \omega \frac{1}{\operatorname{Im} \Omega} \operatorname{Im} \int_{P_{0}}^{Z_{r}} \omega\right] .
$$

Here $E(z, w)$ is the prime form, $\omega$ is the canonical basis of the holomorphic abelian differentials and $\Omega$ is the period matrix of the surface. ${ }^{1}$ The base point $P_{0}$ is arbitrary. For notational convenience, we introduce

$$
g(z, w) \equiv \ln E(z, w)-2 \pi i \int_{P_{0}}^{z} \omega \frac{1}{\operatorname{Im} \Omega} \operatorname{Im} \int_{P_{0}}^{w} \omega
$$

so that (2.7) can be expressed as

$$
\rho(z)=\sum_{r=1}^{N} \alpha_{r} g\left(z, Z_{r}\right) .
$$

Notice that $g(z, w)$ is a function of $z$ and not $\bar{z}$, but that of both of $w$ and $\bar{w}$.

$\rho(z)$ coincides with the coordinate on the light-cone diagram defined as follows. A light-cone diagram consists of cylinders which correspond to propagators of closed strings. On each cylinder, one can introduce a complex coordinate $\rho$ whose real part coincides with the Wick rotated light-cone time $i X^{+}$and imaginary part parametrizes the closed string

\footnotetext{
${ }^{1}$ For the mathematical background relevant for string perturbation theory, we refer the reader to [13].
} 
at each time. The $\rho$ 's on the cylinders are smoothly connected except at the interaction points and we get a complex coordinate $\rho$ on $\Sigma$. $\rho$ is not a good coordinate around the punctures and the interaction points on the light-cone diagram. The interaction points $z=z_{I}(I=1, \cdots, 2 g-2+N)$ are characterized by the equation

$$
\partial \rho\left(z_{I}\right)=0
$$

Since

$$
d s^{2}=-4 \partial X_{\mathrm{cl}}^{+} \bar{\partial} X_{\mathrm{cl}}^{+} d z d \bar{z}=|\partial \rho|^{2} d z d \bar{z}
$$

provides a well-defined metric on the worldsheet except for the points $z=Z_{r}$, $z_{I}$, we can make $\Gamma$ well-defined.

\subsection{Correlation functions on higher genus Riemann surfaces}

As has been demonstrated in [1], all the properties of the worldsheet theory of the longitudinal variables can be deduced from the correlation function of the form

$$
\begin{aligned}
& \left\langle\prod_{r=1}^{N} e^{-i p_{r}^{+} X^{-}}\left(Z_{r} \cdot \bar{Z}_{r}\right) \prod_{s=1}^{M} e^{-i p_{s}^{-} X^{+}}\left(w_{s} \cdot \bar{w}_{s}\right)\right\rangle_{\hat{g}_{z \bar{z}}}^{X^{ \pm}} \\
& \equiv\left(Z^{X}\left[\hat{g}_{z \bar{z}}\right]\right)^{-2} \int\left[d X^{+} d X^{-}\right]_{\hat{g}_{z \bar{z}}} e^{-S^{ \pm}\left[\hat{g}_{z \bar{z}}\right]} \prod_{r=1}^{N} e^{-i p_{r}^{+} X^{-}}\left(Z_{r}, \bar{Z}_{r}\right) \prod_{s=1}^{M} e^{-i p_{s}^{-} X^{+}}\left(w_{s}, \bar{w}_{s}\right) .
\end{aligned}
$$

The correlation functions are normalized by being divided by the factor

$$
\left(Z^{X}\left[\hat{g}_{z \bar{z}}\right]\right)^{2} \equiv\left(\frac{8 \pi^{2} \operatorname{det}^{\prime}\left(-\hat{g}^{z \bar{z}} \partial_{z} \partial_{\bar{z}}\right)}{\int d z \wedge d \bar{z} \sqrt{\hat{g}}}\right)^{-1},
$$

which coincides with the partition function of the worldsheet theory when $d=26$. As is explained in appendix A, taking the integration contours of $X^{ \pm}$appropriately, we can evaluate it and obtain

$$
\begin{aligned}
& \left\langle\prod_{r=1}^{N} e^{-i p_{r}^{+} X^{-}}\left(Z_{r}, \bar{Z}_{r}\right) \prod_{s=1}^{M} e^{-i p_{s}^{-} X^{+}}\left(w_{s}, \bar{w}_{s}\right)\right\rangle_{\hat{g}_{z \bar{z}}}^{X^{ \pm}} \\
& =(2 \pi)^{2} \delta\left(\sum_{s} p_{s}^{-}\right) \delta\left(\sum_{r} p_{r}^{+}\right) \prod_{s} e^{-p_{s}^{-} \frac{\rho+\bar{\rho}}{2}}\left(w_{s}, \bar{w}_{s}\right) e^{-\frac{d-26}{24} \Gamma\left[\hat{g}_{z \bar{z}},-\frac{i}{2}(\rho+\bar{\rho})\right]}
\end{aligned}
$$

Therefore we need to calculate $\Gamma\left[\hat{g}_{z \bar{z}},-\frac{i}{2}(\rho+\bar{\rho})\right]$ to get the correlation function.

Since the metric (2.11) is singular at $z=Z_{r}, z_{I}$ as mentioned above, one gets a divergent result if one naively substitutes $-\frac{i}{2}(\rho+\bar{\rho})$ into $X^{+}$in (2.3). One way to deal with the divergences may be to regularize them as was done in [14]. An alternative way is to integrate the variation formula

$$
\delta(-\Gamma)=\sum_{\mathcal{I}} \delta \mathcal{T}_{\mathcal{I}} \oint_{C_{\mathcal{I}}} \frac{d z}{2 \pi i} \frac{1}{\partial \rho} T^{\text {Liouville }}(z)+\text { c.c. } .
$$




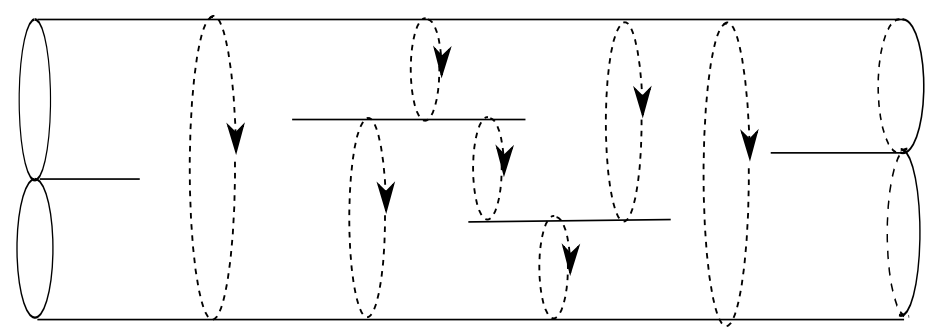

Figure 2. The contours $C_{\mathcal{I}}$.

Here $\mathcal{I}$ labels the internal lines of the light-cone diagram $\Sigma$ and $C_{\mathcal{I}}$ denotes the contour going around it as depicted in figure 2. $\mathcal{T}_{\mathcal{I}}$ is defined as

$$
\mathcal{T}_{\mathcal{I}}=T_{\mathcal{I}}+i \alpha_{\mathcal{I}} \theta_{\mathcal{I}}
$$

where $T_{\mathcal{I}}$ denotes the length of the $\mathcal{I}$-th internal line and $\alpha_{\mathcal{I}}, \theta_{\mathcal{I}}$ denote the string-length and the twist angle for the propagator. $\delta \mathcal{T}_{\mathcal{I}}$ 's and $\delta \overline{\mathcal{T}}_{\mathcal{I}}$ 's should satisfy some linear constraints so that the variation corresponds to that of the shape of a light-cone diagram. $T^{\text {Liouville }}(z)$ denotes the energy-momentum tensor corresponding to the Liouville action (2.3) given as

$$
T^{\text {Liouville }}(z)=(\partial \phi(z))^{2}-2\left(\partial-\partial \ln \hat{g}_{z \bar{z}}\right) \partial \phi(z),
$$

where $\phi$ is now given as

$$
\phi=\ln |\partial \rho|^{2}-\ln \left(2 \hat{g}_{z \bar{z}}\right) .
$$

It is possible to calculate the right hand side of (2.15) and integrate it with respect to the variation to get $\Gamma$. By doing so, we can fix the form of $\Gamma$ as a function of the parameter $\mathcal{T}_{\mathcal{I}}$ 's. Imposing the factorization conditions in the limit where some of the $\mathcal{T}_{\mathcal{I}}$ 's become infinity, it is possible to fix $\Gamma$ completely. By this method, we can calculate $\Gamma$ without encountering divergent constants.

Such a computation was performed in [12] and we can evaluate $\Gamma$ by using the results. The energy-momentum tensor (2.17) with $\phi$ in (2.18) can be rewritten as

$$
\begin{aligned}
T^{\text {Liouville }} & =\left(\partial \ln |\partial \rho|^{2}\right)^{2}-2 \partial^{2} \ln |\partial \rho|^{2}-\left(\partial \ln \hat{g}_{z \bar{z}}\right)^{2}+2 \partial^{2} \ln \hat{g}_{z \bar{z}} \\
& =-2\{\rho, z\}-\left(\left(\partial \ln \hat{g}_{z \bar{z}}\right)^{2}-2 \partial^{2} \ln \hat{g}_{z \bar{z}}\right)
\end{aligned}
$$

where

$$
\{\rho, z\}=\frac{\partial^{3} \rho}{\partial \rho}-\frac{3}{2}\left(\frac{\partial^{2} \rho}{\partial \rho}\right)^{2}
$$

is the Schwarzian derivative. In [12], we have calculated $Z^{\mathrm{LC}}$ which satisfies

$$
\delta \ln Z^{\mathrm{LC}}=\sum_{\mathcal{I}} \delta \mathcal{T}_{\mathcal{I}} \oint_{C_{\mathcal{I}}} \frac{d z}{2 \pi i} \frac{1}{\partial \rho(z)}\left(24\left\langle T^{X}(z)\right\rangle-2\{\rho, z\}\right)+\text { c.c. },
$$


where $\left\langle T^{X}(z)\right\rangle$ denotes the expectation value of the energy-momentum tensor of a free boson $X$. On the other hand, the partition function $Z^{X}\left[\hat{g}_{z \bar{z}}\right]$ satisfies

$$
\delta \ln Z^{X}\left[\hat{g}_{z \bar{z}}\right]=\sum_{\mathcal{I}} \delta \mathcal{T}_{\mathcal{I}} \oint_{C_{\mathcal{I}}} \frac{d z}{2 \pi i} \frac{1}{\partial \rho}\left(\left\langle T^{X}(z)\right\rangle+\frac{1}{24}\left(\left(\partial \ln \hat{g}_{z \bar{z}}\right)^{2}-2 \partial^{2} \ln \hat{g}_{z \bar{z}}\right)\right)+\text { c.c. . }
$$

Comparing (2.21), (2.22) and (2.15), we get

$$
e^{-\Gamma} \propto Z^{\mathrm{LC}}\left(Z^{X}\right)^{-24}
$$

up to a possibly divergent multiplicative factor.

Taking $\hat{g}_{z \bar{z}}$ to be the Arakelov metric $g_{z \bar{z}}^{\mathrm{A}}, Z^{X}\left[\hat{g}_{z \bar{z}}\right]$ was calculated in $[15-20]$ and its explicit form is

$$
Z^{X}\left[g_{z \bar{z}}^{\mathrm{A}}\right]^{24}=e^{c_{g}} e^{2 \delta(\Sigma)},
$$

where $\delta(\Sigma)$ is the Faltings' invariant [21] defined by

$$
\begin{aligned}
e^{-\frac{1}{4} \delta(\Sigma)}= & (\operatorname{det} \operatorname{Im} \Omega)^{\frac{3}{2}}|\theta[\zeta](0 \mid \Omega)|^{2} \frac{\prod_{i=1}^{g}\left(2 g_{\hat{z}_{i} \bar{z}_{i}}^{\mathrm{A}}\right)}{\left|\operatorname{det} \omega_{j}\left(\hat{z}_{i}\right)\right|^{2}} \\
& \times \exp \left[-\sum_{i<j} G^{\mathrm{A}}\left(\hat{z}_{i} ; \hat{z}_{j}\right)+\sum_{i} G^{\mathrm{A}}\left(\hat{z}_{i} ; \hat{w}\right)\right],
\end{aligned}
$$

and $c_{g}$ is a numerical constant which depends on $g$. Here $\hat{z}_{i}(i=1, \ldots, g)$ and $\hat{w}$ are arbitrary points on $\Sigma$, and

$$
\zeta \equiv \sum_{i=1}^{g} \int_{P_{0}}^{\hat{z}_{i}} \omega-\int_{P_{0}}^{\hat{w}} \omega-\Delta .
$$

$\Delta$ denotes the vector of Riemann constants for $P_{0}$. The definitions of the Arakelov metric $g_{z \bar{z}}^{\mathrm{A}}$ and the Arakelov Green's function $G^{\mathrm{A}}(z ; w)$ are given in appendix B. Also taking $\hat{g}_{z \bar{z}}$ to be the Arakelov metric, we obtain [12]

$$
Z^{\mathrm{LC}}=\frac{1}{\left(32 \pi^{2}\right)^{4 h}} e^{2 \delta(\Sigma)} e^{-W} \prod_{r} e^{-2 \operatorname{Re} \bar{N}_{00}^{r r}} \prod_{I}\left|\partial^{2} \rho\left(z_{I}\right)\right|^{-3},
$$

where

$$
\begin{aligned}
-W \equiv & -2 \sum_{I<J} G^{\mathrm{A}}\left(z_{I} ; z_{J}\right)-2 \sum_{r<s} G^{\mathrm{A}}\left(Z_{r} ; Z_{s}\right)+2 \sum_{I, r} G^{\mathrm{A}}\left(z_{I} ; Z_{r}\right) \\
& -\sum_{r} \ln \left(2 g_{Z_{r} \bar{Z}_{r}}^{\mathrm{A}}\right)+3 \sum_{I} \ln \left(2 g_{z_{I} \bar{z}_{I}}^{\mathrm{A}}\right) .
\end{aligned}
$$

$\bar{N}_{00}^{r r}$ denotes one of the Neumann coefficients and is given by

$$
\begin{aligned}
\bar{N}_{00}^{r r} & \equiv \lim _{z \rightarrow Z_{r}}\left[\frac{\rho\left(z_{I^{(r)}}\right)-\rho(z)}{\alpha_{r}}+\ln \left(z-Z_{r}\right)\right] \\
& =\frac{\rho\left(z_{I^{(r)}}\right)}{\alpha_{r}}-\sum_{s \neq r} \frac{\alpha_{s}}{\alpha_{r}} \ln E\left(Z_{r}, Z_{s}\right)+\frac{2 \pi i}{\alpha_{r}} \int_{P_{0}}^{Z_{r}} \omega \frac{1}{\operatorname{Im} \Omega} \sum_{s=1}^{N} \alpha_{s} \operatorname{Im} \int_{P_{0}}^{Z_{s}} \omega,
\end{aligned}
$$


and $z_{I^{(r)}}$ denotes the coordinate of the interaction point at which the $r$-th external line interacts. Therefore we get

$$
\begin{aligned}
e^{-\Gamma} & \propto Z^{\mathrm{LC}} e^{-2 \delta(\Sigma)} \\
& =\frac{1}{\left(32 \pi^{2}\right)^{4 g}} e^{-W} \prod_{r} e^{-2 \operatorname{Re} \bar{N}_{00}^{r r}} \prod_{I}\left|\partial^{2} \rho\left(z_{I}\right)\right|^{-3},
\end{aligned}
$$

and fix the right hand side of (2.14) to be

$$
\begin{aligned}
& \left\langle\prod_{r=1}^{N} e^{-i p_{r}^{+} X^{-}}\left(Z_{r}, \bar{Z}_{r}\right) \prod_{s=1}^{M} e^{-i p_{s}^{-} X^{+}}\left(w_{s}, \bar{w}_{s}\right)\right\rangle_{\hat{g}_{z \bar{z}}}^{X^{ \pm}} \\
& =(2 \pi)^{2} \delta\left(\sum_{s} p_{s}^{-}\right) \delta\left(\sum_{r} p_{r}^{+}\right) \prod_{s} e^{-p_{s}^{-} \frac{\rho+\bar{\rho}}{2}}\left(w_{s}, \bar{w}_{s}\right) \\
& \quad \times\left(\frac{1}{\left(32 \pi^{2}\right)^{4 g}} e^{-W} \prod_{r} e^{-2 \operatorname{Re} \bar{N}_{00}^{r r}} \prod_{I}\left|\partial^{2} \rho\left(z_{I}\right)\right|^{-3}\right)^{\frac{d-26}{24}} .
\end{aligned}
$$

Once we know the correlation function of the form (2.31), it is possible to calculate other correlation functions by differentiating it with respect to $p_{r}^{+}, p_{s}^{-}$. For example,

$$
\begin{aligned}
& \left\langle\partial X^{+}(w) \prod_{r=1}^{N} e^{-i p_{r}^{+} X^{-}}\left(Z_{r}, \bar{Z}_{r}\right) \prod_{s=1}^{M} e^{-i p_{s}^{-} X^{+}}\left(w_{s}, \bar{w}_{s}\right)\right\rangle_{\hat{g}_{z \bar{z}}}^{X^{ \pm}} \\
& =\left.i \partial_{w_{0}} \partial_{p_{0}^{-}}\left\langle\prod_{r=1}^{N} e^{-i p_{r}^{+} X^{-}}\left(Z_{r}, \bar{Z}_{r}\right) \prod_{s=0}^{M+1} e^{-i p_{s}^{-} X^{+}}\left(w_{s}, \bar{w}_{s}\right)\right\rangle_{\hat{g}_{z \bar{z}}}\right|_{p_{0}^{-}=0, w_{0}=w},
\end{aligned}
$$

where we take $p_{M+1}^{-}=-p_{0}^{-}$. From $(2.31)$, we get

$$
\begin{aligned}
& \left\langle\partial X^{+}(w) \prod_{r=1}^{N} e^{-i p_{r}^{+} X^{-}}\left(Z_{r}, \bar{Z}_{r}\right) \prod_{s=1}^{M} e^{-i p_{s}^{-} X^{+}}\left(w_{s}, \bar{w}_{s}\right)\right\rangle_{\hat{g}_{z \bar{z}}}^{X^{ \pm}} \\
& =-\frac{i}{2} \partial \rho(w)\left\langle\prod_{r=1}^{N} e^{-i p_{r}^{+} X^{-}}\left(Z_{r}, \bar{Z}_{r}\right) \prod_{s=1}^{M} e^{-i p_{s}^{-} X^{+}}\left(w_{s}, \bar{w}_{s}\right)\right\rangle_{\hat{g}_{z \bar{z}}}^{X^{ \pm}}
\end{aligned}
$$

It is easy to see that for any functional $F\left[X^{+}\right]$that can be expressed in terms of the derivatives of $X^{+}$and the Fourier modes $e^{-i p X^{+}}$satisfying

$$
F\left[X^{+}+c\right]=F\left[X^{+}\right] \quad(c=\text { const. }),
$$

the following equation holds,

$$
\begin{aligned}
& \left\langle F\left[X^{+}\right] \prod_{r=1}^{N} e^{-i p_{r}^{+} X^{-}}\left(Z_{r}, \bar{Z}_{r}\right) \prod_{s=1}^{M} e^{-i p_{s}^{-} X^{+}}\left(w_{s}, \bar{w}_{s}\right)\right\rangle_{\hat{g}_{z \bar{z}}}^{X^{ \pm}} \\
& =F\left[-\frac{i}{2}(\rho+\bar{\rho})\right]\left\langle\prod_{r=1}^{N} e^{-i p_{r}^{+} X^{-}}\left(Z_{r}, \bar{Z}_{r}\right) \prod_{s=1}^{M} e^{-i p_{s}^{-} X^{+}}\left(w_{s}, \bar{w}_{s}\right)\right\rangle_{\hat{g}_{z \bar{z}}}^{X^{ \pm}} .
\end{aligned}
$$

This implies that the expectation value of $X^{+}(z, \bar{z})$ is equal to $-\frac{i}{2}(\rho(z)+\bar{\rho}(\bar{z}))$. 
The correlation functions involving $X^{-}$can be evaluated in the same way. For example,

$$
\begin{aligned}
& \left\langle\partial X^{-}(z) \prod_{r=1}^{N} e^{-i p_{r}^{+} X^{-}}\left(Z_{r}, \bar{Z}_{r}\right) \prod_{s=1}^{M} e^{-i p_{s}^{-} X^{+}}\left(w_{s}, \bar{w}_{s}\right)\right\rangle_{\hat{g}_{z \bar{z}}}^{X^{ \pm}} \\
& =\left.i \partial_{Z_{0}} \partial_{p_{0}^{+}}\left\langle\prod_{r=0}^{N+1} e^{-i p_{r}^{+} X^{-}}\left(Z_{r}, \bar{Z}_{r}\right) \prod_{s=1}^{M} e^{-i p_{s}^{-} X^{+}}\left(w_{s}, \bar{w}_{s}\right)\right\rangle_{\hat{g}_{z \bar{z}}}\right|_{p_{0}^{+}=0, Z_{0}=z},
\end{aligned}
$$

with $p_{N+1}^{+}=-p_{0}^{+}$and we get from (2.31)

$$
\begin{aligned}
& \left\langle\partial X^{-}(z) \prod_{r=1}^{N} e^{-i p_{r}^{+} X^{-}}\left(Z_{r}, \bar{Z}_{r}\right) \prod_{s=1}^{M} e^{-i p_{s}^{-} X^{+}}\left(w_{s}, \bar{w}_{s}\right)\right\rangle_{\hat{g}_{z \bar{z}}}^{X^{ \pm}} \\
& =\left[\sum_{s=1}^{M}\left(-i p_{s}^{-}\right) \partial_{z} g\left(z, w_{s}\right)+\left.i \partial_{Z_{0}} \partial_{p_{0}^{+}}\left(-\frac{d-26}{24} \Gamma\left[\hat{g}_{z \bar{z}},-\frac{i}{2}\left(\rho^{\prime}+\bar{\rho}^{\prime}\right)\right]\right)\right|_{p_{0}^{+}=0, Z_{0}=z}\right] \\
& \quad \times\left\langle\prod_{r=1}^{N} e^{-i p_{r}^{+} X^{-}}\left(Z_{r}, \bar{Z}_{r}\right) \prod_{s=1}^{M} e^{-i p_{s}^{-} X^{+}}\left(w_{s}, \bar{w}_{s}\right)\right\rangle_{\hat{g}_{z \bar{z}}}^{X^{ \pm}}
\end{aligned}
$$

Here we have introduced

$$
\begin{aligned}
\rho^{\prime}(z) & \equiv \sum_{r=0}^{N+1} \alpha_{r} g\left(z, Z_{r}\right) \\
& =\rho(z)+\alpha_{0}\left(g\left(z, Z_{0}\right)-g\left(z, Z_{N+1}\right)\right) .
\end{aligned}
$$

(2.37) can be rewritten as

$$
\begin{aligned}
& \left\langle\partial X^{-}(z) \prod_{r=1}^{N} e^{-i p_{r}^{+} X^{-}}\left(Z_{r}, \bar{Z}_{r}\right) \prod_{s=1}^{M} e^{-i p_{s}^{-} X^{+}}\left(w_{s}, \bar{w}_{s}\right)\right\rangle_{\hat{g}_{z \bar{z}}}^{X^{ \pm}} \\
& =\left[\sum_{s=1}^{M}\left(-i p_{s}^{-}\right) \partial X^{-(z) X^{+}}\left(w_{s}\right)+\left\langle\partial X^{-}(z)\right\rangle_{\rho}\right] \\
& \times\left\langle\prod_{r=1}^{N} e^{-i p_{r}^{+} X^{-}}\left(Z_{r}, \bar{Z}_{r}\right) \prod_{s=1}^{M} e^{-i p_{s}^{-} X^{+}}\left(w_{s}, \bar{w}_{s}\right)\right\rangle_{\hat{g}_{z \bar{z}}}^{X^{ \pm}},
\end{aligned}
$$

where

$$
\begin{aligned}
\partial X^{-(z) X^{+}}\left(w_{s}\right) & =\partial_{z} g\left(z, w_{s}\right) \\
\left\langle\partial X^{-}(z)\right\rangle_{\rho} & =\left.i \partial_{Z_{0}} \partial_{p_{0}^{+}}\left(-\frac{d-26}{24} \Gamma\left[\hat{g}_{z \bar{z}},-\frac{i}{2}\left(\rho^{\prime}+\bar{\rho}^{\prime}\right)\right]\right)\right|_{p_{0}^{+}=0, Z_{0}=z}
\end{aligned}
$$

$\left\langle\partial X^{-}(z)\right\rangle_{\rho}$ can be formally written as

$$
\left\langle\partial X^{-}(z)\right\rangle_{\rho}=\frac{\left\langle\partial X^{-}(z) \prod_{r=1}^{N} e^{-i p_{r}^{+} X^{-}}\left(Z_{r}, \bar{Z}_{r}\right)\right\rangle_{\hat{g}_{z \bar{z}}}^{X^{ \pm}}}{\left\langle\prod_{r=1}^{N} e^{-i p_{r}^{+} X^{-}}\left(Z_{r}, \bar{Z}_{r}\right)\right\rangle_{\hat{g}_{z \bar{z}}}^{X^{ \pm}}}
$$


We can go ahead and calculate the correlation function involving two $\partial X^{-}$'s as

$$
\begin{aligned}
& \left\langle\partial X^{-}\left(Z_{0}\right) \partial X^{-}(z) \prod_{r=1}^{N} e^{-i p_{r}^{+} X^{-}}\left(Z_{r}, \bar{Z}_{r}\right) \prod_{s=1}^{M} e^{-i p_{s}^{-} X^{+}}\left(w_{s}, \bar{w}_{s}\right)\right\rangle_{\hat{g}_{z \bar{z}}}^{X^{ \pm}} \\
& =\left.i \partial_{Z_{0}} \partial_{p_{0}^{+}}\left\langle\partial X^{-}(z) \prod_{r=0}^{N+1} e^{-i p_{r}^{+} X^{-}}\left(Z_{r}, \bar{Z}_{r}\right) \prod_{s=1}^{M} e^{-i p_{s}^{-} X^{+}}\left(w_{s}, \bar{w}_{s}\right)\right\rangle_{\hat{g}_{z \bar{z}}}^{X_{p_{0}^{+}=0}}\right|_{g^{+}} \\
& =\left[\left(\sum_{s=1}^{M}\left(-i p_{s}^{-}\right) \partial \widetilde{X^{-}\left(Z_{0}\right) X^{+}}\left(w_{s}\right)+\left\langle\partial X^{-}\left(Z_{0}\right)\right\rangle_{\rho}\right)\right. \\
& \times\left(\sum_{s=1}^{M}\left(-i p_{s}^{-}\right) \partial X^{-(z) X^{+}}\left(w_{s}\right)+\left\langle\partial X^{-}(z)\right\rangle_{\rho}\right) \\
& \left.+\left\langle\partial X^{-}\left(Z_{0}\right) \partial X^{-}(z)\right\rangle_{\rho}^{\mathrm{c}}\right] \\
& \times\left\langle\prod_{r=1}^{N} e^{-i p_{r}^{+} X^{-}}\left(Z_{r}, \bar{Z}_{r}\right) \prod_{s=1}^{M} e^{-i p_{s}^{-} X^{+}}\left(w_{s}, \bar{w}_{s}\right)\right\rangle_{\hat{g}_{z \bar{z}}}^{X^{ \pm}}
\end{aligned}
$$

where

$$
\left\langle\partial X^{-}\left(Z_{0}\right) \partial X^{-}(z)\right\rangle_{\rho}^{\mathrm{c}}=\left.i \partial_{Z_{0}} \partial_{p_{0}^{+}}\left(\left\langle\partial X^{-}(z)\right\rangle_{\rho^{\prime}}\right)\right|_{p_{0}^{+}=0},
$$

which can be formally written as

$$
\begin{aligned}
& \left\langle\partial X^{-}\left(Z_{0}\right) \partial X^{-}(z)\right\rangle_{\rho}^{\mathrm{c}} \\
& =\frac{\left\langle\partial X^{-}\left(Z_{0}\right) \partial X^{-}(z) \prod_{r=1}^{N} e^{-i p_{r}^{+} X^{-}}\left(Z_{r}, \bar{Z}_{r}\right)\right\rangle_{\hat{g}_{z \bar{z}}}^{X^{ \pm}}}{\left\langle\prod_{r=1}^{N} e^{-i p_{r}^{+} X^{-}}\left(Z_{r}, \bar{Z}_{r}\right)\right\rangle_{\hat{g}_{z \bar{z}}}^{X^{ \pm}}} \\
& \quad-\frac{\left\langle\partial X^{-}\left(Z_{0}\right) \prod_{r=1}^{N} e^{-i p_{r}^{+} X^{-}}\left(Z_{r}, \bar{Z}_{r}\right)\right\rangle_{\hat{g}_{z \bar{z}}}^{X^{ \pm}}}{\left\langle\prod_{r=1}^{N} e^{-i p_{r}^{+} X^{-}}\left(Z_{r}, \bar{Z}_{r}\right)\right\rangle_{\hat{g}_{z \bar{z}}}^{X^{ \pm}}} \cdot \frac{\left\langle\partial X^{-}(z) \prod_{r=1}^{N} e^{-i p_{r}^{+} X^{-}}\left(Z_{r}, \bar{Z}_{r}\right)\right\rangle_{\hat{g}_{z \bar{z}}}^{X^{ \pm}}}{\left\langle\prod_{r=1}^{N} e^{-i p_{r}^{+} X^{-}}\left(Z_{r}, \bar{Z}_{r}\right)\right\rangle_{\hat{g}_{z \bar{z}}}^{X^{ \pm}}}
\end{aligned}
$$

In this way, it is possible in principle to evaluate all the correlation functions of $X^{+}, X^{-}$ with the source terms $e^{-i p_{r}^{+} X^{-}}\left(Z_{r}, \bar{Z}_{r}\right), e^{-i p_{s}^{-} X^{+}}\left(w_{s}, \bar{w}_{s}\right)$ starting from (2.31). Therefore with the definition of the path integral (A.7) given in appendix A and the anomaly factor $\Gamma$ given in (2.30), we can in principle evaluate all the correlation functions of the worldsheet theory of $X^{ \pm}$.

The worldsheet theory of $X^{ \pm}$thus defined turns out to be a conformal field theory with the central charge $28-d$. It is possible to show that the energy-momentum tensor 
defined as

$$
\begin{aligned}
T^{X^{ \pm}}(z) & =: \partial X^{-}(z) \partial X^{+}(z):-\frac{d-26}{12}\left\{X^{+}, z\right\} \\
& =\lim _{z^{\prime} \rightarrow z}\left(\partial X^{-}\left(z^{\prime}\right) \partial X^{+}(z)-\frac{1}{\left(z^{\prime}-z\right)^{2}}\right)-\frac{d-26}{12}\left\{X^{+}, z\right\},
\end{aligned}
$$

has the following properties:

- $T^{X^{ \pm}}(z)$ is regular at $z=z_{I}$, if there is no operator insertion there.

- The OPE between $T^{X^{ \pm}}(z)$ and $e^{-i p_{r}^{+} X^{-}-i p_{r}^{-} X^{+}}\left(Z_{r}, \bar{Z}_{r}\right)$ is given by

$$
T^{X^{ \pm}}(z) e^{-i p_{r}^{+} X^{-}-i p_{r}^{-} X^{+}}\left(Z_{r}, \bar{Z}_{r}\right) \sim\left[\frac{-p_{r}^{+} p_{r}^{-}}{\left(z-Z_{r}\right)^{2}}+\frac{1}{z-Z_{r}} \frac{\partial}{\partial Z_{r}}\right] e^{-i p_{r}^{+} X^{-}-i p_{r}^{-} X^{+}}\left(Z_{r} . \bar{Z}_{r}\right) .
$$

- The OPE between two $T^{X^{ \pm}}$, s is given by

$$
T^{X^{ \pm}}(z) T^{X^{ \pm}}\left(z^{\prime}\right) \sim \frac{\frac{28-d}{2}}{\left(z-z^{\prime}\right)^{4}}+\frac{1}{\left(z-z^{\prime}\right)^{2}} 2 T^{X^{ \pm}}\left(z^{\prime}\right)+\frac{1}{z-z^{\prime}} \partial T^{X^{ \pm}}\left(z^{\prime}\right) .
$$

These facts can be derived by calculating the expectation value of the energy-momentum tensor and their OPE's as carried out in appendix C.

Therefore the worldsheet theory with the field contents (2.1) becomes a CFT with vanishing central charge. This implies that together with the energy-momentum tensor $T^{\mathrm{LC}}$ of the transverse variables, we can construct a nilpotent BRST operator

$$
Q=\oint \frac{d z}{2 \pi i}\left[c\left(T^{X^{ \pm}}+T^{\mathrm{LC}}\right)+b c \partial c\right]
$$

and obtain a well-defined formulation in the conformal gauge.

\section{Supersymmetric $X^{ \pm}$CFT}

In the case of superstrings [2], it is also possible to consider the theory in noncritical dimensions and construct the conformal gauge formulation corresponding to it. The action of the supersymmetric $X^{ \pm}$CFT is taken to be

$$
S_{\text {super }}^{ \pm}\left[\hat{g}_{z \bar{z}}, \mathcal{X}^{ \pm}\right]=-\frac{1}{2 \pi} \int d^{2} \mathbf{z}\left(\bar{D} \mathcal{X}^{+} D \mathcal{X}^{-}+\bar{D} \mathcal{X}^{-} D \mathcal{X}^{+}\right)+\frac{d-10}{8} \Gamma_{\text {super }}\left[\hat{g}_{z \bar{z}}, \mathcal{X}^{+}\right]
$$

Here the supercoordinate $\mathbf{z}$ is given by

$$
\mathbf{z}=(z, \theta),
$$

where $\theta$ is the Grassmann odd partner of $z$. The superfield $\mathcal{X}^{ \pm}(\mathbf{z}, \overline{\mathbf{z}})$ is defined as

$$
\mathcal{X}^{ \pm}(\mathbf{z}, \overline{\mathbf{z}})=X^{ \pm}(z, \bar{z})+i \theta \psi^{ \pm}(z)+i \bar{\theta} \bar{\psi}^{ \pm}(\bar{z})+\theta \bar{\theta} F^{ \pm}(z, \bar{z}),
$$


$d^{2} \mathbf{z}$ is given by $d^{2} \mathbf{z} \equiv d(\operatorname{Re} z) d(\operatorname{Im} z) d \theta d \bar{\theta}$, and

$$
D \equiv \frac{\partial}{\partial \theta}+\theta \frac{\partial}{\partial z}, \quad \bar{D} \equiv \frac{\partial}{\partial \bar{\theta}}+\bar{\theta} \frac{\partial}{\partial \bar{z}} .
$$

The interaction term $\Gamma_{\text {super }}$ is introduced as

$$
\begin{aligned}
\Gamma_{\text {super }}\left[\hat{g}_{z \bar{z}}, \mathcal{X}^{+}\right] & =-\frac{1}{2 \pi} \int d^{2} \mathbf{z}\left(\bar{D} \Phi D \Phi+\theta \bar{\theta} \hat{g}_{z \bar{z}} \hat{R} \Phi\right) \\
\Phi(\mathbf{z}, \overline{\mathbf{z}}) & =\ln \left(\left(D \Theta^{+}\right)^{2}(\mathbf{z})\left(\bar{D} \bar{\Theta}^{+}\right)^{2}(\overline{\mathbf{z}})\right)-\ln \hat{g}_{z \bar{z}} \\
\Theta^{+}(\mathbf{z}) & =\frac{D \mathcal{X}^{+}}{\left(\partial \mathcal{X}^{+}\right)^{\frac{1}{2}}}(\mathbf{z})
\end{aligned}
$$

which is the super Liouville action defined for variable $\Phi$ with the background metric $d s^{2}=2 \hat{g}_{z \bar{z}} d z d \bar{z}$. In the same way as we have derived (2.14) in the bosonic case, one can deduce

$$
\begin{aligned}
& \left\langle\prod_{r=1}^{N} e^{-i p_{r}^{+} \mathcal{X}^{-}}\left(\mathbf{Z}_{r}, \overline{\mathbf{Z}}_{r}\right) \prod_{s=1}^{M} e^{-i p_{s}^{-} \mathcal{X}^{+}}\left(\mathbf{w}_{s}, \overline{\mathbf{w}}_{s}\right)\right\rangle_{\hat{g}_{z \bar{z}}}^{\mathcal{X}^{ \pm}} \\
& \equiv Z_{\text {super }}^{\mathcal{X}}\left[\hat{g}_{z \bar{z}}\right]^{-2} \int\left[d \mathcal{X}^{+} d \mathcal{X}^{-}\right]_{\hat{g}_{z \bar{z}}} e^{-S_{\text {super }}^{ \pm}\left[\hat{g}_{z \bar{z}}\right]} \prod_{r=1}^{N} e^{-i p_{r}^{+} \mathcal{X}^{-}}\left(\mathbf{Z}_{r}, \overline{\mathbf{Z}}_{r}\right) \prod_{s=1}^{M} e^{-i p_{s}^{-} \mathcal{X}^{+}}\left(\mathbf{w}_{s}, \overline{\mathbf{w}}_{s}\right) \\
& =(2 \pi)^{2} \delta\left(\sum_{s} p_{s}^{-}\right) \delta\left(\sum_{r} p_{r}^{+}\right) \prod_{s} e^{-p_{s}^{-} \frac{\rho+\bar{\rho}}{2}}\left(\mathbf{w}_{s}, \overline{\mathbf{w}}_{s}\right) e^{-\frac{d-10}{8}} \Gamma_{\text {super }}\left[\hat{g}_{z \bar{z}},-\frac{i}{2}(\rho+\bar{\rho})\right]
\end{aligned}
$$

Here

$$
Z_{\text {super }}^{\mathcal{X}}\left[\hat{g}_{z \bar{z}}\right]=\int[d \mathcal{X}]_{\hat{g}_{z \bar{z}}} \exp \left[-\frac{1}{2 \pi} \int d^{2} \mathbf{z} \bar{D} \mathcal{X} D \mathcal{X}\right]
$$

and

$$
\mathbf{Z}_{r}=\left(Z_{r}, \Theta_{r}\right), \quad \mathbf{w}_{s}=\left(w_{s}, \eta_{s}\right) .
$$

$\rho(\mathbf{z})$ which appears on the last line in (3.6) is the supersymmetric version of $\rho(z)$ in (2.7). Its explicit form will be given shortly. Introducing the fermionic partner $\xi$ of $\rho$ given by

$$
\xi \equiv \frac{D \rho}{(\partial \rho)^{\frac{1}{2}}}
$$

we can define the supercoordinate $\boldsymbol{\rho}$ on the light-cone diagram

$$
\boldsymbol{\rho} \equiv(\rho, \xi)
$$

As in the bosonic case, in order to calculate the correlation functions of the CFT, we need to obtain $\Gamma_{\text {super }}\left[\hat{g}_{z \bar{z}},-\frac{i}{2}(\rho+\bar{\rho})\right]$. 


\subsection{Evaluation of $e^{-\Gamma_{\text {super }}}$ on the sphere}

When the worldsheet is a Riemann surface of genus 0 , we can employ the coordinate $z$ on the complex plane to describe the surface. Taking the background metric to be $d s^{2}=d z d \bar{z}$, $\Gamma_{\text {super }}\left[\frac{1}{2},-\frac{i}{2}(\rho+\bar{\rho})\right]$ was derived in [2] by integrating the variation formula

$$
\delta\left(-\Gamma_{\text {super }}\left[\frac{1}{2},-\frac{i}{2}(\rho+\bar{\rho})\right]\right)=\sum_{\mathcal{I}} \delta \mathcal{T}_{\mathcal{I}} \oint_{C_{\mathcal{I}}} \frac{d \mathbf{z}}{2 \pi i} \frac{(-2 S(\mathbf{z}, \boldsymbol{\rho}))}{(D \xi)^{2}}+\text { c.c. }
$$

which is the supersymmetric version of (2.15). Here $S(\mathbf{z}, \boldsymbol{\rho})$ is the super Schwarzian derivative given by

$$
S(\mathbf{z}, \boldsymbol{\rho}) \equiv \frac{D^{4} \xi}{D \xi}-2 \frac{D^{3} \xi D^{2} \xi}{(D \xi)^{2}}
$$

In $[22,23]$, an approach similar to [14] was employed to get $\Gamma_{\text {super }}\left[\frac{1}{2},-\frac{i}{2}(\rho+\bar{\rho})\right]$. Since it is quite tedious to get $\Gamma_{\text {super }}\left[\frac{1}{2},-\frac{i}{2}(\rho+\bar{\rho})\right]$ by these methods, we will look for another approach.

In the sphere case, $\rho(\mathbf{z})$ takes the form

$$
\rho(\mathbf{z})=\sum_{r=1}^{N} \alpha_{r} \ln \left(\mathbf{z}-\mathbf{Z}_{r}\right), \quad \mathbf{z}-\mathbf{Z}_{r} \equiv z-Z_{r}-\theta \Theta_{r} .
$$

This can be written as

$$
\rho(\mathbf{z})=\rho_{b}(z)+\theta f(z)
$$

where

$$
\begin{aligned}
\rho_{b}(z) & =\sum_{r} \alpha_{r} \ln \left(z-Z_{r}\right), \\
f(z) & =-\sum_{r} \frac{\alpha_{r} \Theta_{r}}{z-Z_{r}} .
\end{aligned}
$$

When all the Grassmann parameters $\Theta_{r}$ vanish, the worldsheet theory can be described by an ordinary Riemann surface with coordinate $\rho_{b}$ and obviously

$$
\Gamma_{\text {super }}\left[\hat{g}_{z \bar{z}},-\frac{i}{2}\left(\rho_{b}+\bar{\rho}_{b}\right)\right]=\frac{1}{2} \Gamma\left[\hat{g}_{z \bar{z}},-\frac{i}{2}\left(\rho_{b}+\bar{\rho}_{b}\right)\right]
$$

where $\Gamma\left[\hat{g}_{z \bar{z}},-\frac{i}{2}\left(\rho_{b}+\bar{\rho}_{b}\right)\right]$ is the anomaly factor in the bosonic case given in (2.30). Since $\Gamma\left[\hat{g}_{z \bar{z}},-\frac{i}{2}\left(\rho_{b}+\bar{\rho}_{b}\right)\right]$ is known, the $\Gamma_{\text {super }}\left[\hat{g}_{z \bar{z}},-\frac{i}{2}(\rho+\bar{\rho})\right]$ can be obtained if we can derive how $\Gamma_{\text {super }}\left[\hat{g}_{z \bar{z}},-\frac{i}{2}(\rho+\bar{\rho})\right]$ changes under the deformation

$$
\left(Z_{r}, 0 ; \bar{Z}_{r}, 0\right) \rightarrow\left(Z_{r}, \Theta_{r} ; \bar{Z}_{r}, \bar{\Theta}_{r}\right) \quad(r=1, \cdots, N)
$$

of the moduli parameters.

In the bosonic case, the moduli of the punctured Riemann surfaces can be parametrized either by the coordinates $Z_{r}, \bar{Z}_{r}(r=1, \cdots N)$ of the punctures modulo the $\mathrm{SL}(2, \mathbb{C})$ transformations or by

$$
\mathcal{T}_{\mathcal{I}}^{(b)}=\rho_{b}\left(z_{\mathcal{I}+1}^{(b)}\right)-\rho_{b}\left(z_{\mathcal{I}}^{(b)}\right) \quad(\mathcal{I}=1, \cdots, N-3)
$$


and their complex conjugates $\overline{\mathcal{T}}_{\mathcal{I}}^{(b)}$, where $z_{\mathcal{I}}^{(b)}(\mathcal{I}=1, \cdots N-2)$ are the coordinates of the interaction points which are labeled so that $\mathcal{T}_{\mathcal{I}}^{(b)}$ coincide with $\mathcal{T}_{\mathcal{I}}$ in $(2.16) . \mathcal{T}_{\mathcal{I}}^{(b)}, \overline{\mathcal{T}}_{\mathcal{I}}^{(b)}$ are the natural parameters describing the moduli space from the light-cone gauge point of view and $\Gamma$ is derived by considering how it changes under the variations of them. Therefore in order to calculate $\Gamma_{\text {super }}$, we need a supersymmetric version of (3.18). In [22-24], it is shown that the moduli space of the punctured super Riemann surfaces can be parametrized by $\left(\mathcal{T}_{\mathcal{I}}, \overline{\mathcal{T}}_{\mathcal{I}}, \xi_{I}, \bar{\xi}_{I}\right)$ instead of $\left(Z_{r}, \Theta_{r} ; \bar{Z}_{r}, \bar{\Theta}_{r}\right) . \mathcal{T}_{\mathcal{I}}(\mathcal{I}=1, \cdots, N-3)$ are given as

$$
\mathcal{T}_{\mathcal{I}}=\rho\left(\mathbf{z}_{\mathcal{I}+1}\right)-\rho\left(\mathbf{z}_{\mathcal{I}}\right)
$$

where $\mathbf{z}_{\mathcal{I}}(\mathcal{I}=1, \cdots N-2)$ are the supercoordinates of the interaction points defined in appendix D, and $\overline{\mathcal{T}_{\mathcal{I}}}$ are their complex conjugates. The definitions of the odd supermoduli $\xi_{I}, \bar{\xi}_{I}$ are also given in appendix D.

Equivalence of the parametrizations. Let us demonstrate how the parametrization $\left(Z_{r}, \Theta_{r} ; \bar{Z}_{r}, \bar{\Theta}_{r}\right)$ and $\left(\mathcal{T}_{\mathcal{I}}, \overline{\mathcal{T}}_{\mathcal{I}}, \xi_{I}, \bar{\xi}_{I}\right)$ are related to each other. In order to do so, we consider a tree-level light-cone gauge amplitude for type II superstring theory in 10 dimensions with external lines corresponding to the states

$$
\alpha_{-n_{1}}^{i_{1}} \cdots \bar{\alpha}_{-\bar{n}_{1}}^{\bar{i}_{1}} \cdots \psi_{-s_{1}}^{j_{1}} \cdots \bar{\psi}_{-\bar{s}_{1}}^{\bar{j}_{1}} \cdots|0\rangle_{r} \quad(r=1, \cdots, N) .
$$

The light-cone diagram corresponds to a sphere with $N$ punctures which should be considered as a super Riemann surface. $\rho(\mathbf{z})$ given in (3.13) is the map from the sphere to the light-cone diagram. The amplitude is expressed as an integral of the correlation function

$$
\left\langle V_{1}\left(Z_{1}, \Theta_{1} ; \bar{Z}_{1}, \bar{\Theta}_{1}\right) \cdots V_{N}\left(Z_{N}, \Theta_{N} ; \bar{Z}_{N}, \bar{\Theta}_{N}\right)\right\rangle_{\mathbb{C} \cup \infty}
$$

on the sphere times the anomaly factor

$$
e^{-\Gamma_{\text {super }}\left[\frac{1}{2},-\frac{i}{2}(\rho+\bar{\rho})\right]},
$$

over the moduli parameters. The moduli space of the punctured super Riemann surface is parametrized by the coordinates $\left(Z_{r}, \Theta_{r} ; \bar{Z}_{r}, \bar{\Theta}_{r}\right)(r=1, \ldots, N)$ modded out by the superprojective transformations. Here $V_{r}$ denotes the vertex operator for the state (3.20) and is given by

$$
V_{r}\left(\mathbf{Z}_{r}, \overline{\mathbf{Z}}_{r}\right) \propto \mathcal{A}_{-n_{1}}^{i_{1}} \cdots \overline{\mathcal{A}}_{-\bar{n}_{1}}^{\bar{i}_{1}} \cdots \mathcal{B}_{-s_{1}}^{j_{1}} \cdots \overline{\mathcal{B}}_{-\bar{s}_{1}}^{\bar{j}_{1}} \cdots e^{i \vec{p}_{r} \cdot \overrightarrow{\mathcal{X}}}\left(\mathbf{Z}_{r}, \overline{\mathbf{Z}}_{r}\right) e^{\frac{1}{2} \vec{p}_{r}^{2} \bar{N}_{00}^{r r}}
$$

where

$$
\begin{aligned}
\mathcal{A}_{-n}^{i} & =\oint_{\mathbf{z}_{r}} \frac{d \mathbf{z}}{2 \pi i} i D \mathcal{X}^{i}(\mathbf{z}) e^{-\frac{n}{\alpha_{r}}\left(\rho(\mathbf{z})-\rho\left(\mathbf{z}_{I}(r)\right)\right.}, \\
\mathcal{B}_{-s}^{j} & =\oint_{\mathbf{Z}_{r}} \frac{d \mathbf{z}}{2 \pi i} \frac{D \rho}{(\partial \rho)^{\frac{1}{2}}} i D \mathcal{X}^{j}(\mathbf{z}) e^{-\frac{s}{\alpha_{r}}\left(\rho(\mathbf{z})-\rho\left(\mathbf{z}_{I}(r)\right)\right)}, \\
\bar{N}_{00}^{r r} & =\frac{\rho\left(\tilde{\mathbf{z}}_{I^{(r)}}\right)}{\alpha_{r}}-\sum_{s \neq r} \frac{\alpha_{s}}{\alpha_{r}} \ln \left(\mathbf{Z}_{r}-\mathbf{Z}_{s}\right),
\end{aligned}
$$

$\overline{\mathcal{A}}, \overline{\mathcal{B}}$ are defined in a similar way, and $\mathcal{X}^{i}(\mathbf{z}, \overline{\mathbf{z}})$ are the superfields for the transverse variables. 
It is straightforward to show that $V_{r}\left(Z_{r}, \Theta_{r} ; \bar{Z}_{r}, \bar{\Theta}_{r}\right)$ is expressed as a superconformal transform of $V_{r}\left(Z_{r}, 0 ; \bar{Z}_{r}, 0\right)$ :

$$
U_{r} \bar{U}_{r} V_{r}\left(Z_{r}, 0 ; \bar{Z}_{r}, 0\right)=V_{r}\left(Z_{r}, \Theta_{r} ; \bar{Z}_{r}, \bar{\Theta}_{r}\right) \exp \left(-\operatorname{Re} \frac{\delta \rho\left(\tilde{\mathbf{z}}_{I^{(r)}}\right)}{\alpha_{r}}\right)
$$

with

$$
\begin{aligned}
U_{r} \equiv & \exp \left[\oint_{\left(Z_{r}, 0\right)} \frac{d \mathbf{z}}{2 \pi i} v\left(T^{\mathrm{LC}}(\mathbf{z})-2 S\left(\mathbf{z}, \boldsymbol{\rho}_{b}\right)\right)\right] \\
& \times \exp \left[\oint_{\left(Z_{r}, 0\right)} \frac{d \mathbf{z}}{2 \pi i} \frac{\delta \rho\left(\tilde{\mathbf{z}}_{I^{(r)}}\right)}{\partial \rho_{b}(z)}\left(T^{\mathrm{LC}}(\mathbf{z})-2 S\left(\mathbf{z}, \boldsymbol{\rho}_{b}\right)\right)\right] .
\end{aligned}
$$

Here $T^{\mathrm{LC}}(\mathbf{z})=T_{B}^{\mathrm{LC}}(z)+\theta T_{F}^{\mathrm{LC}}(z)$ is the super energy-momentum tensor in the transverse directions,

$$
\begin{aligned}
v(z, \theta) & =-2 \theta \frac{f(z)}{\partial \rho_{b}(z)} \\
\delta \rho\left(\tilde{\mathbf{z}}_{I}\right) & =\rho\left(\tilde{\mathbf{z}}_{I}\right)-\rho_{b}\left(z_{I}^{(b)}\right), \\
\tilde{\mathbf{z}}_{I} & =\left(z_{I}^{(b)}, \tilde{\theta}_{I}\right)=\left(z_{I}^{(b)},-\frac{\partial f}{\partial^{2} \rho_{b}}\left(z_{I}^{(b)}\right)\right), \\
\boldsymbol{\rho}_{b}(\mathbf{z}) & =\left(\rho_{b}, \theta\left(\partial \rho_{b}\right)^{\frac{1}{2}}\right) \\
S\left(\mathbf{z}, \boldsymbol{\rho}_{b}\right) & =\theta\left(\frac{\partial^{3} \rho_{b}}{2 \partial \rho_{b}}-\frac{3}{4}\left(\frac{\partial^{2} \rho_{b}}{\partial \rho_{b}}\right)^{2}\right),
\end{aligned}
$$

and $z_{I}^{(b)}$ is the coordinate of an interaction point which satisfies $\partial \rho_{b}\left(z_{I}^{(b)}\right)=0$. Indeed, defining

$$
\begin{aligned}
\mathcal{A}_{-n}^{i(b)} & =\oint_{\mathbf{Z}_{r}} \frac{d \mathbf{z}}{2 \pi i} i D \mathcal{X}^{i}(\mathbf{z}) e^{-\frac{n}{\alpha_{r}}\left(\rho_{b}(z)-\rho_{b}\left(z_{I^{(r)}}^{(b)}\right)\right)} \\
\mathcal{B}_{-s}^{j(b)} & =\oint_{\mathbf{Z}_{r}} \frac{d \mathbf{z}}{2 \pi i} \frac{D \rho_{b}}{\left(\partial \rho_{b}\right)^{\frac{1}{2}}} i D \mathcal{X}^{j}(\mathbf{z}) e^{-\frac{s}{\alpha_{r}}\left(\rho_{b}(z)-\rho_{b}\left(z_{I^{(r)}}^{(b)}\right)\right)},
\end{aligned}
$$

it is straightforward to show

$$
U_{r} \mathcal{A}_{-n}^{i(b)} U_{r}^{-1}=\mathcal{A}_{-n}^{i}, \quad U_{r} \mathcal{B}_{-s}^{j(b)} U_{r}^{-1}=\mathcal{B}_{-s}^{j},
$$

and

$$
U_{r} e^{i \vec{p}_{r} \cdot \overrightarrow{\mathcal{X}}}\left(Z_{r}, 0 ; \bar{Z}_{r}, 0\right) e^{\frac{1}{2} \vec{p}_{r}^{2} \bar{N}_{00}^{r r(b)}}=e^{i \vec{p}_{r} \cdot \overrightarrow{\mathcal{X}}}\left(\mathbf{Z}_{r}, \overline{\mathbf{Z}}_{r}\right) \exp \left(\frac{1}{2} \vec{p}_{r}^{2} \bar{N}_{00}^{r r}-\frac{1}{2} \frac{\delta \rho\left(\tilde{\mathbf{z}}_{I}^{(r)}\right)}{\alpha_{r}}\right),
$$

where

$$
\bar{N}_{00}^{r r(b)}=\frac{\rho_{b}\left(z_{I^{(r)}}^{(b)}\right)}{\alpha_{r}}-\sum_{s \neq r} \frac{\alpha_{s}}{\alpha_{r}} \ln \left(Z_{r}-Z_{s}\right) .
$$

It is easy to get (3.25) from these. 
Substituting (3.25) into (3.21), the correlation function (3.21) can be expressed as

$$
\left\langle\prod_{r}\left[U_{r} \bar{U}_{r} V_{r}\left(Z_{r}, 0 ; \bar{Z}_{r}, 0\right)\right]\right\rangle_{\mathbb{C} \cup \infty} \exp \left[\sum_{r} \operatorname{Re} \frac{\delta \rho\left(\tilde{\mathbf{z}}_{\left.I^{(r)}\right)}\right.}{\alpha_{r}}\right] .
$$

Deforming the contours of the the integrals in the exponent of $U_{r}$ in (3.26), we get

$$
\left\langle\prod_{r}\left[U_{r} \bar{U}_{r} V_{r}\left(Z_{r}, 0 ; \bar{Z}_{r}, 0\right)\right]\right\rangle_{\mathbb{C} \cup \infty}=\left\langle\prod_{I}\left(U_{I} \bar{U}_{I}\right) \prod_{\mathcal{I}}\left(U_{\mathcal{I}} \bar{U}_{\mathcal{I}}\right) \prod_{r} V_{r}\left(Z_{r}, 0 ; \bar{Z}_{r}, 0\right)\right\rangle_{\mathbb{C} \cup \infty},
$$

where

$$
\begin{aligned}
U_{I}= & \exp \left[-\oint_{\left(z_{I}^{(b)}, 0\right)} \frac{d \mathbf{z}}{2 \pi i} \frac{\delta \rho\left(\tilde{\mathbf{z}}_{I}\right)}{\partial \rho_{b}(z)}\left(T^{\mathrm{LC}}(\mathbf{z})-2 S\left(\mathbf{z}, \boldsymbol{\rho}_{b}\right)\right)\right] \\
& \times \exp \left[-\oint_{\left(z_{I}^{(b)}, 0\right)} \frac{d \mathbf{z}}{2 \pi i} v\left(T^{\mathrm{LC}}(\mathbf{z})-2 S\left(\mathbf{z}, \boldsymbol{\rho}_{b}\right)\right)\right], \\
U_{\mathcal{I}}= & \exp \left[\delta \mathcal{T}_{\mathcal{I}} \oint_{C_{\mathcal{I}}} \frac{d \mathbf{z}}{2 \pi i} \frac{1}{\partial \rho_{b}(z)}\left(T^{\mathrm{LC}}(\mathbf{z})-2 S\left(\mathbf{z}, \boldsymbol{\rho}_{b}\right)\right)\right] .
\end{aligned}
$$

Using the OPE's

$$
\begin{aligned}
& T_{F}^{\mathrm{LC}}(z) T_{F}^{\mathrm{LC}}(w) \sim \frac{2}{(z-w)^{3}}+\frac{1}{z-w} \frac{1}{2} T_{B}^{\mathrm{LC}}(w), \\
& T_{B}^{\mathrm{LC}}(z) T_{B}^{\mathrm{LC}}(w) \sim \frac{6}{(z-w)^{4}}+\frac{2}{(z-w)^{2}} T_{B}^{\mathrm{LC}}(w)+\frac{1}{z-w} \partial T_{B}^{\mathrm{LC}}(w), \\
& T_{B}^{\mathrm{LC}}(z) T_{F}^{\mathrm{LC}}(w) \sim \frac{\frac{3}{2}}{(z-w)^{2}} T_{F}^{\mathrm{LC}}(w)+\frac{1}{z-w} \partial T_{F}^{\mathrm{LC}}(w),
\end{aligned}
$$

it is straightforward to get

$$
\begin{aligned}
U_{I}= & \exp \left(\xi_{I}\left(\partial^{2} \rho_{b}\right)^{-\frac{3}{4}} T_{F}^{\mathrm{LC}}\left(z_{I}^{(b)}\right)\right) \\
\times & {\left[1+\left(\frac{5}{12} \frac{\partial^{4} \rho_{b}}{\left(\partial^{2} \rho_{b}\right)^{3}}-\frac{3}{4} \frac{\left(\partial^{3} \rho_{b}\right)^{2}}{\left(\partial^{2} \rho_{b}\right)^{4}}\right) \partial f f\right.} \\
& \left.\quad-\frac{2}{3} \frac{\partial^{3} f f}{\left(\partial^{2} \rho_{b}\right)^{2}}+\frac{\partial^{3} \rho_{b}}{\left(\partial^{2} \rho_{b}\right)^{3}} \partial^{2} f f+\frac{1}{12} \frac{\partial^{3} f \partial^{2} f \partial f f}{\left(\partial^{2} \rho_{b}\right)^{4}}\right]\left(z_{I}^{(b)}\right) .
\end{aligned}
$$

Therefore the correlation function (3.21) is expressed as

$$
\begin{aligned}
\left\langle V_{1}\left(Z_{1}, \Theta_{1} ; \bar{Z}_{1}, \bar{\Theta}_{1}\right) \cdots V_{N}\left(Z_{N}, \Theta_{N} ; \bar{Z}_{N}, \bar{\Theta}_{N}\right)\right\rangle_{\mathbb{C} \cup \infty} \\
=\left\langle\prod_{I} \exp \left(\xi_{I}\left(\partial^{2} \rho_{b}\right)^{-\frac{3}{4}} T_{F}^{L C}\left(z_{I}^{(b)}\right)+\text { c.c. }\right) \prod_{\mathcal{I}}\left(U_{\mathcal{I}} \bar{U}_{\mathcal{I}}\right) \prod_{r} V_{r}\left(Z_{r}, 0 ; \bar{Z}_{r}, 0\right)\right\rangle_{\mathbb{C} \cup \infty} \\
\times \exp \left[\sum_{r} \operatorname{Re} \frac{\delta \rho\left(\tilde{\mathbf{z}}_{I(r)}\right)}{\alpha_{r}}\right] \\
\times \prod_{I} \mid\left[1+\left(\frac{5}{12} \frac{\partial^{4} \rho_{b}}{\left(\partial^{2} \rho_{b}\right)^{3}}-\frac{3}{4} \frac{\left(\partial^{3} \rho_{b}\right)^{2}}{\left(\partial^{2} \rho_{b}\right)^{4}}\right) \partial f f\right. \\
\left.\quad-\frac{2}{3} \frac{\partial^{3} f f}{\left(\partial^{2} \rho_{b}\right)^{2}}+\frac{\partial^{3} \rho_{b}}{\left(\partial^{2} \rho_{b}\right)^{3}} \partial^{2} f f+\frac{1}{12} \frac{\partial^{3} f \partial^{2} f \partial f f}{\left(\partial^{2} \rho_{b}\right)^{4}}\right]\left.\left(z_{I}^{(b)}\right)\right|^{2} .
\end{aligned}
$$


From (3.37), we can see that the variation (3.17) of the moduli parameters can be realized by the insertions of operators

$$
\exp \left(\xi_{I}\left(\partial^{2} \rho_{b}\right)^{-\frac{3}{4}} T_{F}^{\mathrm{LC}}\left(z_{I}^{(b)}\right)+\text { c.c. }\right)
$$

and

$$
U_{\mathcal{I}} \bar{U}_{\mathcal{I}}
$$

(3.38) is exactly the superconformal transformation which induces the odd moduli $\xi_{I}, \bar{\xi}_{I}[22-24]$. The insertions of $T_{F}^{\mathrm{LC}} \bar{T}_{F}^{\mathrm{LC}}$ at the interaction points in light-cone gauge perturbation theory arise by integrating superspace correlation function over the odd moduli parameters $\xi_{I}, \bar{\xi}_{I}$. (3.39) corresponds to the shift of $\mathcal{T}_{\mathcal{I}}$ and $\overline{\mathcal{T}}_{\mathcal{I}}$ because of the variation (3.17). Hence the parameters $\mathcal{T}_{\mathcal{I}}, \overline{\mathcal{T}}_{\mathcal{I}}, \xi_{I}, \bar{\xi}_{I}$ parametrize the punctured super Riemann surface and the variations of these parameters are implemented by the insertions of the operators (3.38) and (3.39).

Derivation of $\boldsymbol{\Gamma}_{\text {super. }}$. It is now possible to evaluate $\Gamma_{\text {super }}$. In terms of the parametrization $\left(\mathcal{T}_{\mathcal{I}}, \overline{\mathcal{T}}_{\mathcal{I}}, \xi_{I}, \bar{\xi}_{I}\right)$, the variation $(3.17)$ corresponds to

$$
\left(\mathcal{T}_{\mathcal{I}}^{(b)}, \overline{\mathcal{T}}_{\mathcal{I}}^{(b)}, 0,0\right) \rightarrow\left(\mathcal{T}_{\mathcal{I}}, \overline{\mathcal{T}}_{\mathcal{I}}, \xi_{I}, \bar{\xi}_{I}\right)
$$

From the discussion above, we can see that such a variation can be implemented by inserting operators (3.38) and (3.39). Therefore, starting from the partition function (3.16), we get

$$
\begin{aligned}
& \exp \left(-\Gamma_{\text {super }}\left[\frac{1}{2},-\frac{i}{2}(\rho+\bar{\rho})\right]\right) \\
& =\exp \left(-\frac{1}{2} \Gamma\left[\frac{1}{2},-\frac{i}{2}\left(\rho_{b}+\bar{\rho}_{b}\right)\right]\right) \\
& \quad \times\left\langle\prod_{I} \exp \left(\xi_{I}\left(\partial^{2} \rho_{b}\right)^{-\frac{3}{4}} T_{F}^{\mathrm{LC}}\left(z_{I}^{(b)}\right)+\text { c.c. }\right) \prod_{\mathcal{I}}\left(U_{\mathcal{I}} \bar{U}_{\mathcal{I}}\right)\right\rangle_{\mathbb{C} \cup \infty} .
\end{aligned}
$$

The second factor on the right hand side can easily be evaluated from (3.37) with $V_{r}=1$ and we get the expression

$$
\begin{aligned}
& \exp \left(-\Gamma_{\text {super }}\left[\frac{1}{2},-\frac{i}{2}(\rho+\bar{\rho})\right]\right) \\
& =\exp \left(-\frac{1}{2} \Gamma\left[\frac{1}{2},-\frac{i}{2}\left(\rho_{b}+\bar{\rho}_{b}\right)\right]\right) \exp \left[-\sum_{r} \operatorname{Re} \frac{\delta \rho\left(\tilde{\mathbf{z}}_{I^{(r)}}\right)}{\alpha_{r}}\right] \\
& \times \prod_{I} \mid\left[1+\left(\frac{5}{12} \frac{\partial^{4} \rho_{b}}{\left(\partial^{2} \rho_{b}\right)^{3}}-\frac{3}{4} \frac{\left(\partial^{3} \rho_{b}\right)^{2}}{\left(\partial^{2} \rho_{b}\right)^{4}}\right) \partial f f\right. \\
& \left.\quad-\frac{2}{3} \frac{\partial^{3} f f}{\left(\partial^{2} \rho_{b}\right)^{2}}+\frac{\partial^{3} \rho_{b}}{\left(\partial^{2} \rho_{b}\right)^{3}} \partial^{2} f f+\frac{1}{12} \frac{\partial^{3} f \partial^{2} f \partial f f}{\left(\partial^{2} \rho_{b}\right)^{4}}\right]\left.^{-1}\left(z_{I}^{(b)}\right)\right|^{2} .
\end{aligned}
$$

Since

$$
\delta \rho\left(\tilde{\mathbf{z}}_{I}\right)=-\frac{\partial f f}{\partial^{2} \rho_{b}}\left(z_{I}^{(b)}\right),
$$


we can further rewrite (3.42) as

$$
\begin{aligned}
& \exp \left(-\Gamma_{\text {super }}\left[\frac{1}{2},-\frac{i}{2}(\rho+\bar{\rho})\right]\right) \\
& =\exp \left(-\frac{1}{2} \Gamma\left[\frac{1}{2},-\frac{i}{2}\left(\rho_{b}+\bar{\rho}_{b}\right)\right]-\sum_{r} \Delta \Gamma_{r}-\sum_{I} \Delta \Gamma_{I}\right)
\end{aligned}
$$

where

$$
\begin{aligned}
-\Delta \Gamma_{r}= & \frac{1}{2 \alpha_{r}} \frac{\partial f f}{\partial^{2} \rho_{b}}\left(z_{I^{(r)}}^{(b)}\right)+\text { c.c. }, \\
-\Delta \Gamma_{I}= & \left\{-\left(\frac{5}{12} \frac{\partial^{4} \rho_{b}}{\left(\partial^{2} \rho_{b}\right)^{3}}-\frac{3}{4} \frac{\left(\partial^{3} \rho_{b}\right)^{2}}{\left(\partial^{2} \rho_{b}\right)^{4}}\right) \partial f f+\frac{2}{3} \frac{\partial^{3} f f}{\left(\partial^{2} \rho_{b}\right)^{2}}\right. \\
& \left.-\frac{\partial^{3} \rho_{b}}{\left(\partial^{2} \rho_{b}\right)^{3}} \partial^{2} f f-\frac{1}{12} \frac{\partial^{3} f \partial^{2} f \partial f f}{\left(\partial^{2} \rho_{b}\right)^{4}}\right\}\left(z_{I}^{(b)}\right)+\text { c.c. }
\end{aligned}
$$

In this case, (3.42) can be further simplified using the superspace variables. Indeed, substituting

$$
\exp \left(-\frac{1}{2} \Gamma\left[\frac{1}{2},-\frac{i}{2}\left(\rho_{b}+\bar{\rho}_{b}\right)\right]\right)=\left|\sum_{r} \alpha_{r} Z_{r}\right|^{2} \prod_{I}\left|\partial^{2} \rho_{b}\left(z_{I}^{(b)}\right)\right|^{-\frac{1}{2}} \prod_{r} e^{-\operatorname{Re} \bar{N}_{00}^{r r(b)}}
$$

one can show

$$
\begin{aligned}
& \exp \left(-\Gamma_{\text {super }}\left[\frac{1}{2},-\frac{i}{2}(\rho+\bar{\rho})\right]\right) \\
& =\left|\sum_{r} \alpha_{r} Z_{r}\right|^{2} \exp \left[-\sum_{r} \operatorname{Re}\left(\bar{N}_{00}^{r r(b)}+\frac{\delta \rho\left(\tilde{\mathbf{z}}_{I}(r)\right.}{\alpha_{r}}\right)\right] \prod_{I}\left|\partial^{2} \rho_{b}\left(z_{I}^{(b)}\right)\right|^{-\frac{1}{2}} \\
& \times \prod_{I} \mid\left[1+\left(\frac{5}{12} \frac{\partial^{4} \rho_{b}}{\left(\partial^{2} \rho_{b}\right)^{3}}-\frac{3}{4} \frac{\left(\partial^{3} \rho_{b}\right)^{2}}{\left(\partial^{2} \rho_{b}\right)^{4}}\right) \partial f f\right. \\
& \left.\quad-\frac{2}{3} \frac{\partial^{3} f f}{\left(\partial^{2} \rho_{b}\right)^{2}}+\frac{\partial^{3} \rho_{b}}{\left(\partial^{2} \rho_{b}\right)^{3}} \partial^{2} f f+\frac{1}{12} \frac{\partial^{3} f \partial^{2} f \partial f f}{\left(\partial^{2} \rho_{b}\right)^{4}}\right]\left.^{-1}\left(z_{I}^{(b)}\right)\right|^{2} \\
& =\left|\sum_{r} \alpha_{r} Z_{r}\right|^{2} \exp \left[-\sum_{r} \operatorname{Re}\left(\bar{N}_{00}^{r r(b)}+\frac{\delta \rho\left(\tilde{\mathbf{z}}_{I}(r)\right)}{\alpha_{r}}\right)\right] \\
& \quad \times \prod_{I}\left|\left(\partial^{2} \rho-\frac{5}{3} \frac{\partial^{3} D \rho D \rho}{\partial^{2} \rho}+3 \frac{\partial^{3} \rho \partial^{2} D \rho D \rho}{\left(\partial^{2} \rho\right)^{2}}\right)\left(\tilde{\mathbf{z}}_{I}\right)\right| \\
& \quad \times \exp \left(\frac{1}{2} \sum_{I} \operatorname{Re}\left[\frac{\partial^{3} f f}{\left(\partial^{2} \rho_{b}\right)^{2}}+\frac{\partial^{2} f \partial f}{\left(\partial^{2} \rho_{b}\right)^{2}}-\frac{\partial^{3} \rho_{b}}{\left(\partial^{2} \rho_{b}\right)^{3}} \partial^{2} f f\right]\left(z_{I}^{(b)}\right)\right) .
\end{aligned}
$$


The exponent in the last factor can be given in a more explicit form as

$$
\begin{aligned}
& \sum_{I}\left[\frac{\partial^{3} f f}{\left(\partial^{2} \rho_{b}\right)^{2}}+\frac{\partial^{2} f \partial f}{\left(\partial^{2} \rho_{b}\right)^{2}}-\frac{\partial^{3} \rho_{b}}{\left(\partial^{2} \rho_{b}\right)^{3}} \partial^{2} f f\right]\left(z_{I}^{(b)}\right) \\
& =\sum_{I} \oint_{z_{I}^{(b)}} \frac{d z}{2 \pi i} \frac{\partial^{2} f f}{\left(\partial \rho_{b}\right)^{2}}(z) \\
& =-\sum_{r} \oint_{Z_{r}} \frac{d z}{2 \pi i} \frac{\partial^{2} f f}{\left(\partial \rho_{b}\right)^{2}}(z)-\oint_{\infty} \frac{d z}{2 \pi i} \frac{\partial^{2} f f}{\left(\partial \rho_{b}\right)^{2}}(z) \\
& =-\sum_{r} \frac{2 \Theta_{r}}{\alpha_{r}} \sum_{s \neq r} \frac{\alpha_{s} \Theta_{s}}{Z_{r}-Z_{s}}-\frac{4 \sum_{r} \alpha_{r} \Theta_{r} \sum_{s} \alpha_{s} \Theta_{s} Z_{s}}{\left(\sum_{t} \alpha_{t} Z_{t}\right)^{2}} .
\end{aligned}
$$

Notice that the integrand in the second line is not a one-form. Therefore in deforming the contour in the third line, we should take account of the contribution from $z=\infty$, around which we need to introduce $w=\frac{1}{z}$ as a good coordinate. Substituting (3.48) into (3.47), we eventually obtain

$$
\begin{aligned}
& \exp \left(-\Gamma_{\text {super }}\left[\frac{1}{2},-\frac{i}{2}(\rho+\bar{\rho})\right]\right) \\
& =\left|\sum_{r} \alpha_{r} Z_{r}-\frac{\sum_{r} \alpha_{r} \Theta_{r} \sum_{s} \alpha_{s} \Theta_{s} Z_{s}}{\sum_{t} \alpha_{t} Z_{t}}\right|^{2} \exp \left[-\sum_{r} \operatorname{Re} \bar{N}_{00}^{r r}\right] \\
& \quad \times \prod_{I}\left|\left(\partial^{2} \rho-\frac{5}{3} \frac{\partial^{3} D \rho D \rho}{\partial^{2} \rho}+3 \frac{\partial^{3} \rho \partial^{2} D \rho D \rho}{\left(\partial^{2} \rho\right)^{2}}\right)\left(\tilde{\mathbf{z}}_{I}\right)\right|^{-\frac{1}{2}} .
\end{aligned}
$$

Here we have used

$$
\bar{N}_{00}^{r r}=\bar{N}_{00}^{r r(b)}+\frac{\delta \rho\left(\tilde{\mathbf{z}}_{I^{(r)}}\right)}{\alpha_{r}}+\frac{\Theta_{r}}{\alpha_{r}} \sum_{s \neq r} \frac{\alpha_{s} \Theta_{s}}{Z_{r}-Z_{s}} .
$$

The result (3.49) coincides with the one from the calculations in [2, 22, 23].

\section{$3.2 e^{-\Gamma_{\text {super }}}$ for higher genus Riemann surfaces}

Let us now consider $e^{-\Gamma_{\text {super }}}$ on a higher genus Riemann surface $\Sigma$. All that we need to evaluate (3.6) is the partition function with Grassmann odd parameters $\Theta_{r} \neq 0 .{ }^{2} \rho(\mathbf{z})$ is now given by

$$
\begin{aligned}
\rho(\mathbf{z}) & =\rho_{b}(z)+\theta f(z) \\
& =\sum_{r=1}^{N} \alpha_{r} g\left(\mathbf{z}, \mathbf{Z}_{r}\right),
\end{aligned}
$$

where

$$
\begin{gathered}
f(z)=-\sum_{r} \alpha_{r} \Theta_{r} S_{\delta}\left(z, Z_{r}\right), \\
g\left(\mathbf{z}, \mathbf{z}^{\prime}\right) \equiv g\left(z, z^{\prime}\right)-\theta \theta^{\prime} S_{\delta}\left(z, z^{\prime}\right) .
\end{gathered}
$$

\footnotetext{
${ }^{2}$ In the higher genus case, $\Theta_{r}$ 's, i.e. the Grassmann odd components of the punctures are not enough to parametrize the Grassmann odd directions of the supermoduli space. Therefore what we evaluate here is the partition function on a submanifold in the supermoduli space.
} 
$g\left(z, z^{\prime}\right)$ is defined in $(2.8)$ and $S_{\delta}\left(z, z^{\prime}\right)$ is the Green's function of the worldsheet fermions of the spin structure $\delta$. Here we deal with the case in which all the external lines are in the NS-NS sector and $\delta$ is an even spin structure. $S_{\delta}(z, w)$ is therefore equal to the so-called Szego kernel

$$
S_{\delta}(z, w)=\frac{1}{E(z, w)} \frac{\vartheta[\delta](z-w, \Omega)}{\vartheta[\delta](0, \Omega)} .
$$

In the same way as in the sphere case, we can derive

$$
\begin{aligned}
\left\langle V_{1}\left(Z_{1}, \Theta_{1} ; \bar{Z}_{1}, \bar{\Theta}_{1}\right) \cdots V_{N}\left(Z_{N}, \Theta_{N} ; \bar{Z}_{N}, \bar{\Theta}_{N}\right)\right\rangle_{\Sigma} \\
=\left\langle\prod_{I} \exp \left(\xi_{I}\left(\partial^{2} \rho_{b}\right)^{-\frac{3}{4}} T_{F}^{\mathrm{LC}}\left(z_{I}^{(b)}\right)+\text { c.c. }\right) \prod_{\mathcal{I}}\left(U_{\mathcal{I}} \bar{U}_{\mathcal{I}}\right) \prod_{r} V_{r}\left(Z_{r}, 0 ; \bar{Z}_{r}, 0\right)\right\rangle_{\Sigma} \\
\times \exp \left[\sum_{r} \operatorname{Re} \frac{\delta \rho\left(\tilde{\mathbf{z}}_{I}(r)\right)}{\alpha_{r}}\right] \\
\times \prod_{I} \mid\left[1+\left(\frac{5}{12} \frac{\partial^{4} \rho_{b}}{\left(\partial^{2} \rho_{b}\right)^{3}}-\frac{3}{4} \frac{\left(\partial^{3} \rho_{b}\right)^{2}}{\left(\partial^{2} \rho_{b}\right)^{4}}\right) \partial f f\right. \\
\left.\quad-\frac{2}{3} \frac{\partial^{3} f f}{\left(\partial^{2} \rho_{b}\right)^{2}}+\frac{\partial^{3} \rho_{b}}{\left(\partial^{2} \rho_{b}\right)^{3}} \partial^{2} f f+\frac{1}{12} \frac{\partial^{3} f \partial^{2} f \partial f f}{\left(\partial^{2} \rho_{b}\right)^{4}}\right]\left.\left(z_{I}^{(b)}\right)\right|^{2} .
\end{aligned}
$$

We also have

$$
\begin{aligned}
& \exp \left(-\Gamma_{\text {super }}\left[\hat{g}_{z \bar{z}},-\frac{i}{2}(\rho+\bar{\rho})\right]\right) \\
& =\exp \left(-\frac{1}{2} \Gamma\left[\hat{g}_{z \bar{z}},-\frac{i}{2}\left(\rho_{b}+\bar{\rho}_{b}\right)\right]\right) \\
& \quad \times\left\langle\prod_{I} \exp \left(\xi_{I}\left(\partial^{2} \rho_{b}\right)^{-\frac{3}{4}} T_{F}^{\mathrm{LC}}\left(z_{I}^{(b)}\right)+\text { c.c. }\right) \prod_{\mathcal{I}}\left(U_{\mathcal{I}} \bar{U}_{\mathcal{I}}\right)\right\rangle_{\Sigma},
\end{aligned}
$$

and eventually get the same form as (3.44)

$$
\begin{aligned}
& \exp \left(-\Gamma_{\text {super }}\left[\hat{g}_{z \bar{z}},-\frac{i}{2}(\rho+\bar{\rho})\right]\right) \\
& =\exp \left(-\frac{1}{2} \Gamma\left[\hat{g}_{z \bar{z}},-\frac{i}{2}\left(\rho_{b}+\bar{\rho}_{b}\right)\right]-\sum_{r} \Delta \Gamma_{r}-\sum_{I} \Delta \Gamma_{I}\right),
\end{aligned}
$$

where

$$
\begin{aligned}
-\Delta \Gamma_{r}= & \frac{1}{2 \alpha_{r}} \frac{\partial f f}{\partial^{2} \rho_{b}}\left(z_{I(r)}^{(b)}\right)+\text { c.c. }, \\
-\Delta \Gamma_{I}= & \left\{-\left(\frac{5}{12} \frac{\partial^{4} \rho_{b}}{\left(\partial^{2} \rho_{b}\right)^{3}}-\frac{3}{4} \frac{\left(\partial^{3} \rho_{b}\right)^{2}}{\left(\partial^{2} \rho_{b}\right)^{4}}\right) \partial f f+\frac{2}{3} \frac{\partial^{3} f f}{\left(\partial^{2} \rho_{b}\right)^{2}}\right. \\
& \left.-\frac{\partial^{3} \rho_{b}}{\left(\partial^{2} \rho_{b}\right)^{3}} \partial^{2} f f-\frac{1}{12} \frac{\partial^{3} f \partial^{2} f \partial f f}{\left(\partial^{2} \rho_{b}\right)^{4}}\right\}\left(z_{I}^{(b)}\right)+\text { c.c. }
\end{aligned}
$$

We do not know a good identity like (3.48) to rewrite this expression further using superspace variables.

The normalization of the correlation function (3.6) is defined by the third line of (3.6) with $e^{-\Gamma_{\text {super }}}$ given in (3.56). 


\subsection{Correlation functions of supersymmetric $X^{ \pm}$CFT}

We can proceed in the same say as in the bosonic case and calculate the correlation functions of supersymmetric $X^{ \pm}$CFT, starting from (3.6). We get equations such as

$$
\begin{aligned}
& \left\langle F\left[\mathcal{X}^{+}\right] \prod_{r=1}^{N} e^{-i p_{r}^{+} \mathcal{X}^{-}}\left(\mathbf{Z}_{r}, \overline{\mathbf{Z}}_{r}\right) \prod_{s=1}^{M} e^{-i p_{s}^{-} \mathcal{X}^{+}}\left(\mathbf{w}_{s}, \overline{\mathbf{w}}_{s}\right)\right\rangle_{\hat{g}_{z \bar{z}}}^{\mathcal{X}^{ \pm}} \\
& =F\left[-\frac{i}{2}(\rho+\bar{\rho})\right]\left\langle\prod_{r=1}^{N} e^{-i p_{r}^{+} \mathcal{X}^{-}}\left(\mathbf{Z}_{r}, \overline{\mathbf{Z}}_{r}\right) \prod_{s=1}^{M} e^{-i p_{s}^{-} \mathcal{X}^{+}}\left(\mathbf{w}_{s}, \overline{\mathbf{w}}_{s}\right)\right\rangle_{\hat{g}_{z \bar{z}}}^{\mathcal{X}^{ \pm}}, \\
& \left\langle D \mathcal{X}^{-}(\mathbf{z}) \prod_{r=1}^{N} e^{-i p_{r}^{+} \mathcal{X}^{-}}\left(\mathbf{Z}_{r}, \overline{\mathbf{Z}}_{r}\right) \prod_{s=1}^{M} e^{-i p_{s}^{-} \mathcal{X}^{+}}\left(\mathbf{w}_{s}, \overline{\mathbf{w}}_{s}\right)\right\rangle_{\hat{g}_{z \bar{z}}}^{\mathcal{X}^{ \pm}}
\end{aligned}
$$

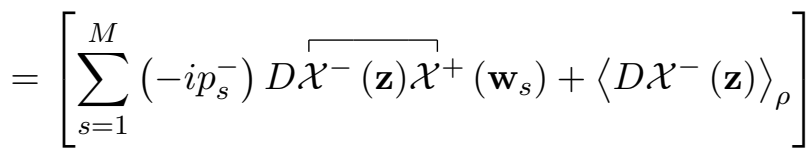

$$
\begin{aligned}
& \times\left\langle\prod_{r=1}^{N} e^{-i p_{r}^{+} \mathcal{X}^{-}}\left(\mathbf{Z}_{r}, \overline{\mathbf{Z}}_{r}\right) \prod_{s=1}^{M} e^{-i p_{s}^{-} \mathcal{X}^{+}}\left(\mathbf{w}_{s}, \overline{\mathbf{w}}_{s}\right)\right\rangle_{\hat{g}_{z \bar{z}}}^{\mathcal{X}^{ \pm}}, \\
& \left\langle D \mathcal{X}^{-}\left(\mathbf{Z}_{0}\right) D \mathcal{X}^{-}(\mathbf{z}) \prod_{r=1}^{N} e^{-i p_{r}^{+} \mathcal{X}^{-}}\left(\mathbf{Z}_{r}, \overline{\mathbf{Z}}_{r}\right) \prod_{s=1}^{M} e^{-i p_{s}^{-} \mathcal{X}^{+}}\left(\mathbf{w}_{s}, \overline{\mathbf{w}}_{s}\right)\right\rangle_{\hat{g}_{z \bar{z}}}^{\mathcal{X}^{ \pm}} \\
& =\left[\left(\sum_{s=1}^{M}\left(-i p_{s}^{-}\right) D \overline{\mathcal{X}^{-}\left(\mathbf{Z}_{\mathbf{0}}\right) \mathcal{X}^{+}}\left(\mathbf{w}_{s}\right)+\left\langle D \mathcal{X}^{-}\left(\mathbf{Z}_{0}\right)\right\rangle_{\rho}\right)\right.
\end{aligned}
$$

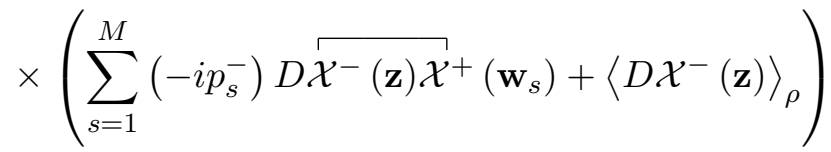

$$
\begin{aligned}
& \left.+\left\langle D \mathcal{X}^{-}\left(\mathbf{Z}_{0}\right) D \mathcal{X}^{-}(\mathbf{z})\right\rangle_{\rho}^{\mathrm{c}}\right] \\
& \times\left\langle\prod_{r=1}^{N} e^{-i p_{r}^{+} \mathcal{X}^{-}}\left(\mathbf{Z}_{r}, \overline{\mathbf{Z}}_{r}\right) \prod_{s=1}^{M} e^{-i p_{s}^{-} \mathcal{X}^{+}}\left(\mathbf{w}_{s}, \overline{\mathbf{w}}_{s}\right)\right\rangle_{\hat{g}_{z \bar{z}}}^{X^{ \pm}}
\end{aligned}
$$

where

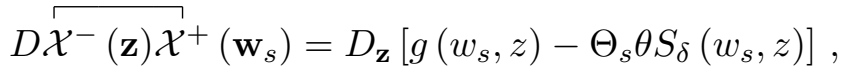

$$
\begin{aligned}
& \left\langle D \mathcal{X}^{-}(z)\right\rangle_{\rho}=\left.i D_{\mathbf{Z}_{0}} \partial_{p_{0}^{+}}\left(-\frac{d-10}{8} \Gamma_{\text {super }}\left[\hat{g}_{z \bar{z}},-\frac{i}{2}(\rho+\bar{\rho})\right]\right)\right|_{p_{0}^{+}=0, \mathbf{Z}_{0}=\mathbf{z}}, \\
& \left\langle D \mathcal{X}^{-}\left(\mathbf{Z}_{0}\right) D \mathcal{X}^{-}(\mathbf{z})\right\rangle_{\rho}^{\mathrm{c}}=\left.i D_{\mathbf{Z}_{0}} \partial_{p_{0}^{+}}\left(\left\langle D \mathcal{X}^{-}(\mathbf{z})\right\rangle_{\rho^{\prime}}\right)\right|_{p_{0}^{+}=0}, \\
& \rho^{\prime}(\mathbf{z})=\rho(\mathbf{z})+\alpha_{0}\left(g\left(z, Z_{0}\right)-\theta \Theta_{0} S_{\delta}\left(z, Z_{0}\right)\right. \\
& \left.-g\left(z, Z_{N+1}\right)+\theta \Theta_{N+1} S_{\delta}\left(z, Z_{N+1}\right)\right) .
\end{aligned}
$$


In this way, it is possible to evaluate all the correlation functions of $\mathcal{X}^{+}, \mathcal{X}^{-}$with the source terms $e^{-i p_{r}^{+} \mathcal{X}^{-}}\left(\mathbf{Z}_{r}, \overline{\mathbf{Z}}_{r}\right), e^{-i p_{s}^{-} \mathcal{X}^{+}}\left(\mathbf{w}_{s}, \overline{\mathbf{w}}_{s}\right)$ starting from (3.6). The worldsheet theory of $\mathcal{X}^{ \pm}$thus defined turns out to be a superconformal field theory with the central charge $\hat{c}=12-d$. The energy-momentum tensor is defined as

$$
\begin{aligned}
T^{\mathcal{X}^{ \pm}}(\mathbf{z})= & \frac{1}{2}: \partial \mathcal{X}^{+} D \mathcal{X}^{-}(\mathbf{z}):+\frac{1}{2}: D \mathcal{X}^{+} \partial \mathcal{X}^{-}(\mathbf{z}):-\frac{d-10}{4} S\left(\mathbf{z}, \mathcal{X}^{+}\right) \\
= & \frac{1}{2} \lim _{\mathbf{w} \rightarrow \mathbf{z}}\left(\partial \mathcal{X}^{+}(\mathbf{w}) D \mathcal{X}^{-}(\mathbf{z})-\partial_{\mathbf{w}} D_{\mathbf{z}} \ln (\mathbf{w}-\mathbf{z})\right) \\
& +\frac{1}{2} \lim _{\mathbf{w} \rightarrow \mathbf{z}}\left(D \mathcal{X}^{+}(\mathbf{w}) \partial \mathcal{X}^{-}(\mathbf{z})-D_{\mathbf{w}} \partial_{\mathbf{z}} \ln (\mathbf{w}-\mathbf{z})\right) \\
& -\frac{d-10}{4} S\left(\mathbf{z}, \mathcal{X}^{+}\right),
\end{aligned}
$$

where

$$
S\left(\mathbf{z}, \mathcal{X}^{+}\right)=\frac{\partial^{2} \Theta^{+}}{D \Theta^{+}}-\frac{2 \partial D \Theta^{+} \partial \Theta^{+}}{\left(D \Theta^{+}\right)^{2}}
$$

and $\Theta^{+}(\mathbf{z})$ is given in (3.5). It is possible to show that this has the following properties:

- $T^{\mathcal{X}^{ \pm}}(\mathbf{z})$ is regular at $\mathbf{z}=\tilde{\mathbf{z}}_{I}$, if there is no operator insertion there.

- The OPE between $T^{\mathcal{X}^{ \pm}}(\mathbf{z})$ and $e^{-i p_{r}^{+} \mathcal{X}^{-}-i p_{r}^{-} \mathcal{X}^{+}}\left(\mathbf{Z}_{r}, \overline{\mathbf{Z}}_{r}\right)$ is given by

$$
\begin{aligned}
& T^{\mathcal{X}^{ \pm}}(\mathbf{z}) e^{-i p_{r}^{+} \mathcal{X}^{-}-i p_{r}^{-} \mathcal{X}^{+}}\left(\mathbf{Z}_{r}, \overline{\mathbf{Z}}_{r}\right) \\
& \sim \frac{\theta-\Theta_{r}}{\left(\mathbf{z}-\mathbf{Z}_{r}\right)^{2}}\left(-p_{r}^{+} p_{r}^{-}\right) e^{-i p_{r}^{+} \mathcal{X}^{-}-i p_{r}^{-} \mathcal{X}^{+}}\left(\mathbf{Z}_{r}, \overline{\mathbf{Z}}_{r}\right) \\
& \quad+\frac{1}{\mathbf{z}-\mathbf{Z}_{r}} \frac{1}{2} D e^{-i p_{r}^{+} \mathcal{X}^{-}-i p_{r}^{-} \mathcal{X}^{+}}\left(\mathbf{Z}_{r}, \overline{\mathbf{Z}}_{r}\right) \\
& \quad+\frac{\theta-\Theta_{r}}{\mathbf{z}-\mathbf{Z}_{r}} \partial e^{-i p_{r}^{+} \mathcal{X}^{-}-i p_{r}^{-} \mathcal{X}^{+}}\left(\mathbf{Z}_{r}, \overline{\mathbf{Z}}_{r}\right) .
\end{aligned}
$$

- The OPE between two $T^{\mathcal{X}^{ \pm}}$'s is given by

$$
\begin{aligned}
& T^{\mathcal{X}^{ \pm}}(\mathbf{z}) T^{\mathcal{X}^{ \pm}}\left(\mathbf{z}^{\prime}\right) \\
& \sim \frac{12-d}{4\left(\mathbf{z}-\mathbf{z}^{\prime}\right)^{3}}+\frac{\theta-\theta^{\prime}}{\left(\mathbf{z}-\mathbf{z}^{\prime}\right)^{2}} \frac{3}{2} T^{\mathcal{X}^{ \pm}}\left(\mathbf{z}^{\prime}\right)+\frac{1}{\mathbf{z}-\mathbf{z}^{\prime}} \frac{1}{2} D T^{\mathcal{X}^{ \pm}}\left(\mathbf{z}^{\prime}\right)+\frac{\theta-\theta^{\prime}}{\mathbf{z}-\mathbf{z}^{\prime}} \partial T^{\mathcal{X}^{ \pm}}\left(\mathbf{z}^{\prime}\right),
\end{aligned}
$$

which corresponds to the super Virasoro algebra with the central charge $\hat{c}=12-d$.

These facts can be derived by calculating the expectation value of the energy-momentum tensor and their OPE's. Some of the details of these calculations are given in appendix E. It follows that combined with the transverse variables $\mathcal{X}^{i}(\mathbf{z}, \overline{\mathbf{z}})(i=1, \ldots, d-2)$, the total central charge of the system becomes $\hat{c}=10$. This implies that with the ghost superfields $B(\mathbf{z})$ and $C(\mathbf{z})$ defined as

$$
B(\mathbf{z})=\beta(z)+\theta b(z), \quad C(\mathbf{z})=c(z)+\theta \gamma(z),
$$

it is possible to construct a nilpotent BRST charge

$$
Q_{\mathrm{B}}=\oint \frac{d \mathbf{z}}{2 \pi i}\left[-C\left(T^{\mathcal{X}^{ \pm}}+T^{\mathrm{LC}}\right)+\left(C \partial C-\frac{1}{4}(D C)^{2}\right) B\right]
$$




\section{Discussions}

In this paper, we have studied the longitudinal part of the worldsheet theory corresponding to the light-cone gauge string field theory in noncritical dimensions, on higher genus Riemann surfaces. We have defined and calculated the correlation functions of both bosonic and supersymmetric cases and shown that they have the right properties to be used to describe the theory in noncritical dimensions.

In order to analyze the supersymmetric case, we have proposed a way to calculate $\Gamma_{\text {super }}$ which is much simpler than those proposed in [22, 23] or [2]. The correlation functions in the higher genus case are quite complicated because we have not been able to find a way to express it in terms of the superfield $\rho(\mathbf{z})$ so far. It seems easy to generalize our method to the $N=2$ case and calculate the anomaly factor on higher genus Riemann surfaces. Such a calculation will be useful for studying the amplitudes involving Ramond sector fields [25] or amplitudes in Green-Schwarz formalism [26-30].

With the $X^{ \pm}$CFT studied in this paper, it is possible to describe the conformal gauge formulation of the light-cone gauge theory in noncritical dimensions. We can construct the nilpotent BRST charge and rewrite the light-cone gauge amplitudes in terms of the conformal gauge worldsheet theory. The amplitudes in noncritical dimensions can be used to regularize various divergences in a gauge invariant way. In [3-6], we have dealt with the contact term divergences of the tree amplitudes. The results in this paper shall be used in analyzing the higher-loop superstring amplitudes in separate publications.

\section{Acknowledgments}

One of the authors (K.M.) would like to acknowledge the hospitality of Particle Theory Group at University of Tsukuba, where part of this work was done. This work was supported in part by Grant-in-Aid for Scientific Research (C) (25400242) and (15K05063) from MEXT.

\section{A Definition of the path integral of $X^{ \pm}$variables}

In this appendix, we explain how to define the path integral on the right hand side of (2.12) following [12]. Since the action of $X^{ \pm}$variables is not bounded below, we need to take the integration contours of $X^{ \pm}$carefully to define the path integral. Let us first recapitulate how we do so in the critical case. The action for $X^{ \pm}$is given as

$$
S_{d=26}^{ \pm}=-\frac{1}{4 \pi} \int d z \wedge d \bar{z} i\left(\partial X^{+} \bar{\partial} X^{-}+\partial X^{-} \bar{\partial} X^{+}\right)
$$

The path integrals to be defined are of the form

$$
\int\left[d X^{+} d X^{-}\right]_{\hat{g}_{z \bar{z}}} e^{-S_{d=26}^{ \pm}} \prod_{r=1}^{N} e^{-i p_{r}^{+} X^{-}}\left(Z_{r}, \bar{Z}_{r}\right) \prod_{s=1}^{M} e^{-i p_{s}^{-} X^{+}}\left(z_{s}, \bar{z}_{s}\right),
$$


which is supposed to be equal to

$$
(2 \pi)^{2} \delta\left(\sum_{r} p_{r}^{+}\right) \delta\left(\sum_{s} p_{s}^{-}\right) \prod_{s=1}^{M} e^{-i p_{s}^{-} X_{\mathrm{cl}}^{+}}\left(z_{s}, \bar{z}_{s}\right) Z^{X}\left[\hat{g}_{z \bar{z}}\right]^{2},
$$

where $X_{\mathrm{cl}}^{ \pm}(z, \bar{z})$ are solutions to the equations of motion with the source terms

$$
\begin{aligned}
& \partial \bar{\partial} X_{\mathrm{cl}}^{+}(z, \bar{z})=-i \sum_{r} p_{r}^{+}(-2 \pi i) \delta^{2}\left(z-Z_{r}\right), \\
& \partial \bar{\partial} X_{\mathrm{cl}}^{-}(z, \bar{z})=-i \sum_{s} p_{s}^{-}(-2 \pi i) \delta^{2}\left(z-z_{s}\right) .
\end{aligned}
$$

In order to derive (A.3), we decompose the variable $X^{ \pm}$as

$$
X^{ \pm}(z, \bar{z})=X_{\mathrm{cl}}^{ \pm}(z, \bar{z})+x^{ \pm}+\delta X^{ \pm}(z, \bar{z})
$$

where $x^{ \pm}+\delta X^{ \pm}(z, \bar{z})$ are the fluctuations around the solutions with

$$
\int d z \wedge d \bar{z} \sqrt{\hat{g}} \delta X^{ \pm}=0 .
$$

Integrals over $X^{ \pm}$are expressed as those over $x^{ \pm}$and $\delta X^{ \pm}$. In (A.2), we take the integration contours of $x^{ \pm}$and $\delta X^{+}-\delta X^{-}$to be along the real axis and that of $\delta X^{+}+\delta X^{-}$to be along the imaginary axis. Then (A.2) becomes well-defined and is evaluated to be (A.3).

Based on this definition of the path integral (A.2), it is possible to evaluate (2.12) as follows. The right hand side of (2.12) can be expanded as

$$
\begin{aligned}
& \left(Z^{X}\left[\hat{g}_{z \bar{z}}\right]\right)^{-2} \int\left[d X^{+} d X^{-}\right]_{\hat{g}_{z \bar{z}}} e^{-S^{ \pm}\left[\hat{g}_{z \bar{z}}\right]} \prod_{r=1}^{N} e^{-i p_{r}^{+} X^{-}}\left(Z_{r}, \bar{Z}_{r}\right) \prod_{s=1}^{M} e^{-i p_{s}^{-} X^{+}}\left(w_{s}, \bar{w}_{s}\right) \\
& =\left(Z^{X}\left[\hat{g}_{z \bar{z}}\right]\right)^{-2} \int\left[d X^{+} d X^{-}\right]_{\hat{g}_{z \bar{z}}} e^{-S_{d=26}^{ \pm}\left[\hat{g}_{z \bar{z}}\right]} \prod_{r=1}^{N} e^{-i p_{r}^{+} X^{-}}\left(Z_{r}, \bar{Z}_{r}\right) \prod_{s=1}^{M} e^{-i p_{s}^{-} X^{+}}\left(w_{s}, \bar{w}_{s}\right) \\
& \quad \times \sum_{n=0}^{\infty} \frac{1}{n !}\left(-\frac{d-26}{24} \Gamma\left[\hat{g}_{z \bar{z}}, X^{+}\right]\right)^{n}
\end{aligned}
$$

and expressed in terms of the correlation functions of the theory in the critical dimensions. Using the prescription for the contours of the integration, it is straightforward to prove

$$
\begin{aligned}
0= & \int\left[d X^{+} d X^{-}\right]_{\hat{g}_{z \bar{z}}} e^{-S_{d=26}^{ \pm}} \\
& \times \prod_{r=1}^{N} e^{-i p_{r}^{+} X^{-}}\left(Z_{r} \cdot \bar{Z}_{r}\right) \prod_{s=1}^{M} e^{-i p_{s}^{-} X^{+}}\left(z_{s} . \bar{z}_{s}\right) \prod_{p=1}^{n} \partial \delta X^{+}\left(z_{p}\right) \prod_{q=1}^{m} \bar{\partial} \delta X^{+}\left(\bar{z}_{q}\right),
\end{aligned}
$$

if $n$ or $m \geq 1$. Using this and (A.3), it is possible to show that the right hand side of (A.7) is equal to

$$
(2 \pi)^{2} \delta\left(\sum_{s} p_{s}^{-}\right) \delta\left(\sum_{r} p_{r}^{+}\right) \prod_{s} e^{-p_{s}^{-} \frac{\rho+\bar{\rho}}{2}}\left(z_{s}, \bar{z}_{s}\right) e^{-\frac{d-26}{24} \Gamma\left[\hat{g}_{z \bar{z}},-\frac{i}{2}(\rho+\bar{\rho})\right]} .
$$




\section{B Arakelov metric and Arakelov Green's function}

In this appendix, we give the definitions of the Arakelov metric and the Arakelov Green's function, following $[17,31]$.

Let us define $\mu_{z \bar{z}}$ as

$$
\mu_{z \bar{z}} \equiv \frac{1}{2 g} \omega(z) \frac{1}{\operatorname{Im} \Omega} \bar{\omega}(\bar{z})
$$

We note that

$$
\int_{\Sigma} d z \wedge d \bar{z} i \mu_{z \bar{z}}=1
$$

which follows from

$$
\int_{\Sigma} \omega_{\mu} \wedge \bar{\omega}_{\nu}=-2 i \operatorname{Im} \Omega_{\mu \nu}
$$

The Arakelov metric on $\Sigma$,

$$
d s_{\mathrm{A}}^{2}=2 g_{z \bar{z}}^{\mathrm{A}} d z d \bar{z},
$$

is defined so that its scalar curvature $R^{\mathrm{A}} \equiv-2 g^{\mathrm{A} z \bar{z}} \partial \bar{\partial} \ln g_{z \bar{z}}^{\mathrm{A}}$ satisfies

$$
g_{z \bar{z}}^{\mathrm{A}} R^{\mathrm{A}}=-8 \pi(g-1) \mu_{z \bar{z}} .
$$

This condition determines $g_{z \bar{z}}^{\mathrm{A}}$ only up to an overall constant, which we will choose later.

The Arakelov Green's function $G^{\mathrm{A}}(z, \bar{z} ; w, \bar{w})$ with respect to the Arakelov metric is defined to satisfy

$$
\begin{aligned}
-\partial_{z} \partial_{\bar{z}} G^{\mathrm{A}}(z, \bar{z} ; w, \bar{w}) & =2 \pi \delta^{2}(z-w)-2 \pi \mu_{z \bar{z}} \\
\int_{\Sigma} d z \wedge d \bar{z} i \mu_{z \bar{z}} G^{\mathrm{A}}(z, \bar{z} ; w, \bar{w}) & =0 .
\end{aligned}
$$

One can obtain a more explicit form of $G^{\mathrm{A}}(z, \bar{z} ; w, \bar{w})$ by solving (B.6) for $G^{\mathrm{A}}(z, \bar{z} ; w, \bar{w})$. Let $F(z, \bar{z} ; w, \bar{w})$ be the $\left(-\frac{1}{2},-\frac{1}{2}\right) \times\left(-\frac{1}{2},-\frac{1}{2}\right)$ form on $\Sigma \times \Sigma$ defined as

$$
F(z, \bar{z} ; w, \bar{w})=\exp \left[-2 \pi \operatorname{Im} \int_{w}^{z} \omega \frac{1}{\operatorname{Im} \Omega} \operatorname{Im} \int_{w}^{z} \omega\right]|E(z, w)|^{2} .
$$

It is easy to show

$$
\partial_{z} \partial_{\bar{z}} \ln F(z, \bar{z} ; w, \bar{w})=-2 \pi i \delta^{2}(z-w)-2 \pi g \mu_{z \bar{z}} .
$$

Putting eqs. (B.8) and (B.5) together, we find that $G^{\mathrm{A}}(z, \bar{z} ; w, \bar{w})$ is given by

$$
G^{\mathrm{A}}(z, \bar{z} ; w, \bar{w})=-\ln F(z, \bar{z} ; w, \bar{w})-\frac{1}{2} \ln \left(2 g_{z \bar{z}}^{\mathrm{A}}\right)-\frac{1}{2} \ln \left(2 g_{w \bar{w}}^{\mathrm{A}}\right),
$$

up to an additive constant independent of $z, \bar{z}$ and $w, \bar{w}$. This possible additive constant can be absorbed into the ambiguity in the overall constant of $g_{z \bar{z}}^{\mathrm{A}}$ mentioned above. It is required that (B.9) holds exactly as it is $[17,18,31]$. This implies that

$$
2 g_{z \bar{z}}^{\mathrm{A}}=\lim _{w \rightarrow z} \exp \left[-G^{\mathrm{A}}(z, \bar{z} ; w, \bar{w})-\ln |z-w|^{2}\right],
$$

and the overall constant of $g_{z \bar{z}}^{\mathrm{A}}$ is, in principle, determined by the second relation in (B.6). 


\section{Calculations of correlation functions of bosonic $X^{ \pm}$CFT}

In this appendix, we calculate the correlation functions involving the energy-momentum tensor of the bosonic $X^{ \pm}$CFT and show various properties of the theory.

\section{C.1 Evaluation of $\left\langle\partial X^{-}(z)\right\rangle_{\rho}$}

We evaluate the relevant quantities starting from the expectation value $\left\langle\partial X^{-}(z)\right\rangle_{\rho}$ which is given as

$$
\left\langle\partial X^{-}(z)\right\rangle_{\rho}=\left.2 i \partial_{Z_{0}} \partial_{\alpha_{0}}\left(-\frac{d-26}{24} \Gamma\left[g_{z \bar{z}}^{\mathrm{A}},-\frac{i}{2}\left(\rho^{\prime}+\bar{\rho}^{\prime}\right)\right]\right)\right|_{\alpha_{0}=0, Z_{0}=z} .
$$

In the following, various quantities defined by using $\rho^{\prime}$ given in (2.38) instead of $\rho$ in (2.7) will be denoted by attaching a prime. We also define

$$
\begin{aligned}
\partial_{Z_{0}} \tilde{g}\left(Z_{0}, Z_{N+1}\right) & \equiv \frac{\partial}{\partial Z_{0}} \ln E\left(Z_{0}, Z_{N+1}\right)-2 \pi i \omega\left(Z_{0}\right) \frac{1}{\operatorname{Im} \Omega} \operatorname{Im} \int_{Z_{0}}^{Z_{N+1}} \omega \\
\partial_{Z_{N+1}} \tilde{g}\left(Z_{N+1}, Z_{0}\right) & \equiv \frac{\partial}{\partial Z_{N+1}} \ln E\left(Z_{0}, Z_{N+1}\right)-2 \pi i \omega\left(Z_{N+1}\right) \frac{1}{\operatorname{Im} \Omega} \operatorname{Im} \int_{Z_{N+1}}^{Z_{0}} \omega,
\end{aligned}
$$

which make the following calculations look simpler.

In order to get $\left\langle\partial X^{-}(z)\right\rangle$, we need the following expansions in terms of $\alpha_{0}$ :

$$
\begin{aligned}
z_{I}^{\prime}-z_{I}= & -\frac{\alpha_{0}}{\partial^{2} \rho\left(z_{I}\right)} \frac{\partial}{\partial z_{I}}\left(g\left(z_{I}, Z_{0}\right)-g\left(z_{I}, Z_{N+1}\right)\right)+\mathcal{O}\left(\alpha_{0}^{2}\right), \\
z_{I^{(0)}}^{\prime}-Z_{0}= & -\frac{\alpha_{0}}{\partial \rho\left(Z_{0}\right)} \\
& -\alpha_{0}^{2}\left(\frac{\partial^{2} \rho\left(Z_{0}\right)}{\left(\partial \rho\left(Z_{0}\right)\right)^{3}}+\frac{1}{\left(\partial \rho\left(Z_{0}\right)\right)^{2}} \partial_{Z_{0}} \tilde{g}\left(Z_{0}, Z_{N+1}\right)\right)+\mathcal{O}\left(\alpha_{0}^{3}\right), \\
z_{I^{(N+1)}}^{\prime}-Z_{N+1}= & \frac{\alpha_{0}}{\partial \rho\left(Z_{N+1}\right)}-\alpha_{0}^{2}\left(\frac{\partial^{2} \rho\left(Z_{N+1}\right)}{\left(\partial \rho\left(Z_{N+1}\right)\right)^{3}}\right. \\
& \left.+\frac{1}{\left(\partial \rho\left(Z_{N+1}\right)\right)^{2}} \partial_{N+1} \tilde{g}\left(Z_{N+1}, Z_{0}\right)\right)+\mathcal{O}\left(\alpha_{0}^{3}\right), \\
\bar{N}_{00}^{\prime r r}= & \bar{N}_{00}^{r r}+\frac{\alpha_{0}}{\alpha_{r}}\left(g\left(z_{I^{(r)}}, Z_{0}\right)-g\left(z_{I^{(r)}}, Z_{N+1}\right)-g\left(Z_{r}, Z_{0}\right)+g\left(Z_{r}, Z_{N+1}\right)\right) \\
& +\mathcal{O}\left(\alpha_{0}^{2}\right), \\
\bar{N}_{00}^{\prime 00}= & \ln \left(-\frac{\alpha_{0}}{\partial \rho\left(Z_{0}\right)}\right)-1+\frac{\alpha_{0}}{2} \frac{\partial^{2} \rho\left(Z_{0}\right)}{\left(\partial \rho\left(Z_{0}\right)\right)^{2}}+\frac{\alpha_{0}}{\partial \rho\left(Z_{0}\right)} \partial_{Z_{0}} \tilde{g}\left(Z_{0}, Z_{N+1}\right)+\mathcal{O}\left(\alpha_{0}^{2}\right), \\
\bar{N}_{00}^{\prime N+1 N+1}= & \ln \left(\frac{\alpha_{0}}{\partial \rho\left(Z_{N+1}\right)}\right)-1 \\
& -\frac{\alpha_{0}}{2} \frac{\partial^{2} \rho\left(Z_{N+1}\right)}{\left(\partial \rho\left(Z_{N+1}\right)\right)^{2}}-\frac{\alpha_{0}}{\partial \rho\left(Z_{N+1}\right)} \partial_{Z_{N+1}} \tilde{g}\left(Z_{N+1}, Z_{0}\right)+\mathcal{O}\left(\alpha_{0}^{2}\right), \\
\partial^{2} \rho^{\prime}\left(z_{I}^{\prime}\right)= & \partial^{2} \rho\left(z_{I}\right)+\alpha_{0} \frac{\partial^{2}}{\partial z_{I}^{2}}\left(g\left(z_{I}, Z_{0}\right)-g\left(z_{I}, Z_{N+1}\right)\right) \\
& -\alpha_{0} \frac{\partial^{3} \rho}{\partial^{2} \rho}\left(z_{I}\right) \frac{\partial}{\partial z_{I}}\left(g\left(z_{I}, Z_{0}\right)-g\left(z_{I}, Z_{N+1}\right)\right)+\mathcal{O}\left(\alpha_{0}^{2}\right),
\end{aligned}
$$




$$
\begin{aligned}
\partial^{2} \rho^{\prime}\left(z_{I^{(0)}}^{\prime}\right)= & -\frac{\left(\partial \rho\left(Z_{0}\right)\right)^{2}}{\alpha_{0}}+3 \partial^{2} \rho\left(Z_{0}\right)+2 \partial \rho\left(Z_{0}\right) \partial_{Z_{0}} \tilde{g}\left(Z_{0}, Z_{N+1}\right)+\mathcal{O}\left(\alpha_{0}\right), \\
\partial^{2} \rho^{\prime}\left(z_{I^{(N+1)}}^{\prime}\right)= & \frac{\left(\partial \rho\left(Z_{N+1}\right)\right)^{2}}{\alpha_{0}}+3 \partial^{2} \rho\left(Z_{N+1}\right)+2 \partial \rho\left(Z_{N+1}\right) \partial_{Z_{N+1}} \tilde{g}\left(Z_{N+1}, Z_{0}\right)+\mathcal{O}\left(\alpha_{0}\right), \\
\partial^{3} \rho^{\prime}\left(z_{I}^{\prime}\right)= & \partial^{3} \rho\left(z_{I}\right)+\mathcal{O}\left(\alpha_{0}\right), \\
\partial^{3} \rho^{\prime}\left(z_{I^{(0)}}^{\prime}\right)= & -\frac{2}{\alpha_{0}^{2}}\left(\partial \rho\left(Z_{0}\right)\right)^{3} \\
& +\frac{6}{\alpha_{0}} \partial \rho\left(Z_{0}\right) \partial^{2} \rho\left(Z_{0}\right)+\frac{6}{\alpha_{0}}\left(\partial \rho\left(Z_{0}\right)\right)^{2} \partial_{Z_{0}} \tilde{g}\left(Z_{0}, Z_{N+1}\right)+\mathcal{O}\left(\alpha_{0}^{0}\right), \\
\partial^{3} \rho^{\prime}\left(z_{I^{(N+1)}}^{\prime}\right)= & -\frac{2}{\alpha_{0}^{2}}\left(\partial \rho\left(Z_{N+1}\right)\right)^{3}-\frac{6}{\alpha_{0}} \partial \rho\left(Z_{N+1}\right) \partial^{2} \rho\left(Z_{N+1}\right) \\
& -\frac{6}{\alpha_{0}}\left(\partial \rho\left(Z_{N+1}\right)\right)^{2} \partial_{Z_{N+1}} \tilde{g}\left(Z_{N+1}, Z_{0}\right)+\mathcal{O}\left(\alpha_{0}^{0}\right) .
\end{aligned}
$$

Here $z_{I}^{\prime}(I=1, \ldots, 2 g-2+N), z_{I^{(0)}}^{\prime}, z_{I^{(N+1)}}^{\prime}$ are the interaction points for $\rho^{\prime}$, which behave as $z_{I}^{\prime} \rightarrow z_{I}, z_{I^{(0)}}^{\prime} \rightarrow Z_{0}, z_{I^{(N+1)}}^{\prime} \rightarrow Z_{N+1}$ in the limit $\alpha_{0} \rightarrow 0$ respectively.

We also need the formula for the variation of $-W^{\prime}$, which is the $-W$ in (2.28) defined for $\rho^{\prime}$. Comparing the behavior of the Schwarzian derivative for $z \sim z_{I}$

$$
-2\{\rho, z\} \sim \frac{3}{\left(z-z_{I}\right)^{2}}+\frac{1}{z-z_{I}} \frac{\partial^{3} \rho\left(z_{I}\right)}{\partial^{2} \rho\left(z_{I}\right)},
$$

derived from $\partial \rho(z) \sim \partial^{2} \rho\left(z_{I}\right)\left(z-z_{I}\right)+\frac{1}{2} \partial^{3} \rho\left(z_{I}\right)\left(z-z_{I}\right)^{2}$ with

$$
-2\{\rho, z\} \sim \frac{3}{\left(z-z_{I}\right)^{2}}+\frac{1}{z-z_{I}} \frac{\partial}{\partial z_{I}}(-W),
$$

which is given in [12] (eq. (B.15)), we obtain the formula

$$
\frac{\partial(-W)}{\partial z_{I}}=\frac{\partial^{3} \rho\left(z_{I}\right)}{\partial^{2} \rho\left(z_{I}\right)}
$$

From this, we get the expansion of $-W^{\prime}$ as

$$
\begin{aligned}
& \frac{\partial\left(-W^{\prime}\right)}{\partial z_{I}^{\prime}}=\frac{\partial(-W)}{\partial z_{I}}+\mathcal{O}\left(\alpha_{0}\right) \\
& \frac{\partial\left(-W^{\prime}\right)}{\partial z_{I^{(0)}}^{\prime}}=\frac{2}{\alpha_{0}} \partial \rho\left(Z_{0}\right)-2 \partial_{Z_{0}} \tilde{g}\left(Z_{0}, Z_{N+1}\right)+\mathcal{O}\left(\alpha_{0}\right) \\
& \frac{\partial\left(-W^{\prime}\right)}{\partial z_{I^{(N+1)}}^{\prime}}=-\frac{2}{\alpha_{0}} \partial \rho\left(Z_{N+1}\right)-2 \partial_{Z_{N+1}} \tilde{g}\left(Z_{N+1}, Z_{0}\right)+\mathcal{O}\left(\alpha_{0}\right) .
\end{aligned}
$$

Using all these, it is straightforward to derive

$$
\begin{aligned}
\left\langle\partial X^{-}(z)\right\rangle_{\rho} & =\left.2 i \partial_{Z_{0}} \partial_{\alpha_{0}}\left(-\frac{d-26}{24} \Gamma\left[g_{z \bar{z}}^{\mathrm{A}},-\frac{i}{2}\left(\rho^{\prime}+\bar{\rho}^{\prime}\right)\right]\right)\right|_{\alpha_{0}=0, Z_{0}=z} \\
& =\left.2 i \partial_{Z_{0}} \partial_{\alpha_{0}}\left[-\frac{d-26}{24}\left(-2 \sum_{r=0}^{N+1} \operatorname{Re} \bar{N}_{00}^{\prime r r}-\frac{3}{2} \sum_{I} \ln \left|\partial^{2} \rho^{\prime}\left(z_{I}^{\prime}\right)\right|^{2}-W^{\prime}\right)\right]\right|_{\alpha_{0}=0, Z_{0}=z} \\
& =\frac{d-26}{24} 2 i \partial_{z}\left[\sum_{r=1}^{N} B_{r}(z, \bar{z})+\sum_{I=1}^{2 g-2+N} B_{I}(z, \bar{z})\right]
\end{aligned}
$$


where

$$
\begin{aligned}
& B_{r}(z, \bar{z}) \equiv \frac{1}{\alpha_{r}}\left(g\left(Z_{r}, z\right)-g\left(z_{I^{(r)}}, z\right)\right)+\text { c.c. } \\
& B_{I}(z, \bar{z}) \equiv \frac{\partial^{3} \rho}{2\left(\partial^{2} \rho\right)^{2}}\left(z_{I}\right) \frac{\partial}{\partial z_{I}} g\left(z_{I}, z\right)-\frac{3}{2 \partial^{2} \rho\left(z_{I}\right)} \frac{\partial^{2}}{\partial z_{I}^{2}} g\left(z_{I}, z\right)+\text { c.c. } .
\end{aligned}
$$

Notice that $\left\langle\partial X^{-}(z)\right\rangle_{\rho}$ is meromorphic with respect to $z$.

\section{C.2 Energy-momentum tensor}

Now let us check if the energy-momentum tensor $T^{X^{ \pm}}$of the $X^{ \pm}$CFT defined in (2.45) satisfies the desired properties.

We first consider the correlation function of the form

$$
\left\langle T^{X^{ \pm}}(z) \prod_{r=1}^{N} e^{-i p_{r}^{+} X^{-}}\left(Z_{r}, \bar{Z}_{r}\right) \prod_{s=1}^{M} e^{-i p_{s}^{-} X^{+}}\left(w_{s}, \bar{w}_{s}\right)\right\rangle_{\hat{g}_{z \bar{z}}}^{X^{ \pm}},
$$

which can be evaluated as

$$
\begin{aligned}
& \left\langle T^{X^{ \pm}}(z) \prod_{r=1}^{N} e^{-i p_{r}^{+} X^{-}}\left(Z_{r}, \bar{Z}_{r}\right) \prod_{s=1}^{M} e^{-i p_{s}^{-} X^{+}}\left(w_{s}, \bar{w}_{s}\right)\right\rangle_{\hat{g}_{z \bar{z}}}^{X^{ \pm}} \\
& =\left[-\frac{i}{2} \partial \rho(z) \sum_{s=1}^{M}\left(-i p_{s}^{-}\right) \partial X^{-}(z) X^{+}\left(w_{s}\right)+\left\langle T^{X^{ \pm}}(z)\right\rangle_{\rho}\right] \\
& \quad \times\left\langle\prod_{r=1}^{N} e^{-i p_{r}^{+} X^{-}}\left(Z_{r}, \bar{Z}_{r}\right) \prod_{s=1}^{M} e^{-i p_{s}^{-} X^{+}}\left(w_{s}, \bar{w}_{s}\right)\right\rangle_{\hat{g}_{z \bar{z}}}^{X^{ \pm}},
\end{aligned}
$$

where

$$
\begin{aligned}
\left\langle T^{X^{ \pm}}(z)\right\rangle_{\rho}= & -\frac{i}{2} \partial \rho(z)\left\langle\partial X^{-}(z)\right\rangle_{\rho}-\frac{d-26}{12}\{\rho, z\} \\
& -\left.\frac{1}{3} \frac{\partial^{3}}{\partial z^{\prime 3}} E\left(z^{\prime}, z\right)\right|_{z^{\prime}=z}-\pi \omega(z) \frac{1}{\operatorname{Im} \Omega} \omega(z) .
\end{aligned}
$$

Since (C.11) can be considered as the generating function of correlation functions with one $T^{X^{ \pm}}(z)$ insertion, we can deduce various properties of $T^{X^{ \pm}}$from it. The first thing we check is whether $T^{X^{ \pm}}(z)$ is singular at $z=z_{I}$. Even if there are no operator insertions at $z=z_{I}$, (C.9) implies that $\partial X^{-}(z)$ is singular there. It will be disastrous for BRST quantization of the worldsheet theory, if $T^{X^{ \pm}}(z)$ is singular at such points. For $z \sim z_{I}$, one finds ${ }^{3}$

$$
\begin{aligned}
\left\langle\partial X^{-}(z)\right\rangle_{\rho} & \sim-\frac{d-26}{24} 2 i\left[\frac{1}{\left(z-z_{I}\right)^{3}} \frac{3}{\partial^{2} \rho\left(z_{I}\right)}-\frac{1}{\left(z-z_{I}\right)^{2}} \frac{1}{2} \frac{\partial^{3} \rho\left(z_{I}\right)}{\left(\partial^{2} \rho\left(z_{I}\right)\right)^{2}}+\sum_{r} \frac{1}{\alpha_{r}} \frac{1}{z-z_{I^{(r)}}}\right], \\
\partial \rho(z) & \sim\left(z-z_{I}\right) \partial^{2} \rho\left(z_{I}\right)+\frac{1}{2}\left(z-z_{I}\right)^{2} \partial^{3} \rho\left(z_{I}\right)+\mathcal{O}\left(\left(z-z_{I}\right)^{3}\right),
\end{aligned}
$$

\footnotetext{
${ }^{3}$ On the right hand side of the first equation in (C.14), the sum $\sum_{r}$ is over the $r$ 's such that $z_{I}=z_{I^{(r)}}$.
} 
and

$$
-\frac{i}{2} \partial \rho(z)\left\langle\partial X^{-}(z)\right\rangle_{\rho} \sim-\frac{d-26}{24}\left[\frac{3}{\left(z-z_{I}\right)^{3}}+\frac{1}{z-z_{I}} \frac{\partial^{3} \rho\left(z_{I}\right)}{\partial^{2} \rho\left(z_{I}\right)}+\mathcal{O}(1)\right] .
$$

With (C.6), (C.12) and (C.13), this implies that $T^{X^{ \pm}}(z)$ is not singular at $z=z_{I}$.

The singular behaviors at $z=Z_{r}$ are obtained as

$$
\begin{aligned}
\left\langle\partial X^{-}(z)\right\rangle_{\rho} \sim & \frac{d-26}{24} 2 i \\
& \times\left[\frac{\frac{1}{\alpha_{r}}}{z-Z_{r}}+\lim _{z \rightarrow Z_{r}}\left(\partial B_{r}(z)-\frac{\frac{1}{\alpha_{r}}}{z-Z_{r}}\right)+\sum_{s \neq r} \partial B_{s}\left(Z_{r}\right)+\sum_{I} \partial B_{I}\left(Z_{r}\right)\right] \\
\partial \rho(z) \sim & \frac{\alpha_{r}}{z-Z_{r}}+\lim _{z \rightarrow Z_{r}}\left(\partial \rho(z)-\frac{\alpha_{r}}{z-Z_{r}}\right)
\end{aligned}
$$

and

$$
\begin{aligned}
& -\frac{i}{2} \partial \rho(z)\left\langle\partial X^{-}(z)\right\rangle_{\rho} \\
& \sim \frac{d-26}{24}\left[\frac{1}{\left(z-Z_{r}\right)^{2}}\right. \\
& \quad+\frac{1}{z-Z_{r}}\left\{\frac{1}{\alpha_{r}} \lim _{z \rightarrow Z_{r}}\left(\partial \rho(z)-\frac{\alpha_{r}}{z-Z_{r}}\right)\right. \\
& \left.\left.\quad+\alpha_{r}\left(\lim _{z \rightarrow Z_{r}}\left(\partial B_{r}(z)-\frac{\frac{1}{\alpha_{r}}}{z-Z_{r}}\right)+\sum_{s \neq r} \partial B_{s}\left(z_{r}\right)+\sum_{I} \partial B_{I}\left(Z_{r}\right)\right)\right\}\right] \\
& \sim \frac{d-26}{24}\left[\frac{1}{\left(z-Z_{r}\right)^{2}}+\frac{1}{z-Z_{r}} \frac{\partial}{\partial Z_{r}}\left(-2 \sum_{s} \operatorname{Re} \bar{N}_{00}^{r r}-\frac{3}{2} \sum_{I} \ln \left|\partial \rho\left(z_{I}\right)\right|^{2}\right)\right. \\
& \left.\quad+\frac{1}{z-Z_{r}} \sum_{I}\left(\frac{\partial z_{I}}{\partial Z_{r}} \frac{\partial(-W)}{\partial z_{I}}+\frac{\partial \bar{z}_{I}}{\partial Z_{r}} \frac{\partial(-W)}{\partial \bar{z}_{I}}\right)\right] .
\end{aligned}
$$

In order to get (C.17), we have used

$$
\begin{aligned}
& \lim _{z \rightarrow Z_{r}}\left(\partial B_{r}(z)-\frac{\frac{1}{\alpha_{r}}}{z-Z_{r}}\right)+\sum_{s \neq r} \partial B_{s}\left(z_{r}\right)+\sum_{I} \partial B_{I}\left(Z_{r}\right) \\
& =-\frac{\pi}{\alpha_{r}} \int_{P_{0}}^{Z_{r}} \omega \frac{1}{\operatorname{Im} \Omega} \omega\left(Z_{r}\right)-\frac{1}{\alpha_{r}} \frac{\partial}{\partial Z_{r}} g\left(z_{I^{(r)}}, Z_{r}\right)+\sum_{s \neq r} \frac{1}{\alpha_{s}} \frac{\partial}{\partial Z_{r}}\left(g\left(Z_{s}, Z_{r}\right)-g\left(z_{I^{(s)}}, Z_{r}\right)\right) \\
& \quad+\sum_{s} \frac{1}{\alpha_{s}} \frac{\partial}{\partial Z_{r}}\left(\bar{g}\left(Z_{s}, Z_{r}\right)-\bar{g}\left(z_{I^{(s)}}, Z_{r}\right)\right)+\sum_{I} \frac{\partial}{\partial Z_{r}} B_{I}\left(Z_{r}\right), \\
& \left.\lim _{z \rightarrow Z_{r}}\left(\partial \rho(z)-\frac{\alpha_{r}}{z-Z_{r}}\right)=\sum_{s \neq r} \alpha_{s} \frac{\partial}{\partial Z_{r}} g\left(Z_{r}, Z_{s}\right)-2 \pi i \alpha_{r} \omega\left(Z_{r}\right) \frac{1}{\operatorname{Im} \Omega} \operatorname{Im} \int_{P_{0}}^{Z_{r}} \omega, \quad \text { (C. } 18\right)
\end{aligned}
$$


and

$$
\begin{aligned}
\frac{\partial z_{I}}{\partial Z_{r}}= & -\frac{\alpha_{r}}{\partial^{2} \rho\left(z_{I}\right)} \partial_{z_{I}} \partial_{Z_{r}} g\left(z_{I}, Z_{r}\right), \\
\frac{\partial \bar{z}_{I}}{\partial Z_{r}}= & -\frac{\alpha_{r}}{\partial^{2} \bar{\rho}\left(\bar{z}_{I}\right)} \partial_{\bar{z}_{I}} \partial_{Z_{r}} \bar{g}\left(\bar{z}_{I}, Z_{r}\right), \\
\frac{\partial \bar{N}_{00}^{r r}}{\partial Z_{r}}= & \frac{\partial}{\partial Z_{r}} g\left(z_{I^{(r)}}, Z_{r}\right)-\sum_{s \neq r} \frac{\alpha_{s}}{\alpha_{r}} \frac{\partial}{\partial Z_{r}} g\left(Z_{r}, Z_{s}\right) \\
& +2 \pi i \omega\left(Z_{r}\right) \frac{1}{\operatorname{Im} \Omega} \operatorname{Im} \int_{P_{0}}^{Z_{r}} \omega+\pi \int_{P_{0}}^{Z_{r}} \omega \frac{1}{\operatorname{Im} \Omega} \omega\left(Z_{r}\right), \\
\frac{\partial \bar{N}_{00}^{s s}}{\partial Z_{r}}= & \frac{\alpha_{r}}{\alpha_{s}} \frac{\partial}{\partial Z_{r}}\left(g\left(z_{I^{(s)}}, Z_{r}\right)-g\left(Z_{s}, Z_{r}\right)\right)(s \neq r), \\
\frac{\partial \bar{N}_{00}^{s s *}}{\partial Z_{r}}= & \frac{\alpha_{r}}{\alpha_{s}} \frac{\partial}{\partial Z_{r}}\left(\bar{g}\left(\bar{z}_{I^{(s)}}, Z_{r}\right)-\bar{g}\left(\bar{Z}_{s}, Z_{r}\right)\right) .
\end{aligned}
$$

Now with

$$
-2\{\rho, z\} \sim-\frac{1}{\left(z-Z_{r}\right)^{2}}+\frac{1}{z-Z_{r}} \frac{\partial(-W)}{\partial Z_{r}},
$$

it is easy to derive

$$
\left\langle T^{X^{ \pm}}(z)\right\rangle_{\rho} \sim \frac{1}{z-Z_{r}} \frac{d}{d Z_{r}}\left(-\frac{d-26}{24} \Gamma\right)
$$

(C.21) implies

$$
T^{X^{ \pm}}(z) e^{-i p_{r}^{+} X^{-}}\left(Z_{r}, \bar{Z}_{r}\right) \sim \frac{1}{z-Z_{r}} \frac{d}{d Z_{r}} e^{-i p_{r}^{+} X^{-}}\left(Z_{r}, \bar{Z}_{r}\right) .
$$

In the same way, the following OPE can be derived:

$$
T^{X^{ \pm}}(z) e^{-i p_{r}^{+} X^{-}-i p_{r}^{-} X^{+}}\left(Z_{r}, \bar{Z}_{r}\right) \sim\left[\frac{-p_{r}^{+} p_{r}^{-}}{\left(z-Z_{r}\right)^{2}}+\frac{1}{z-Z_{r}} \frac{d}{d Z_{r}}\right] e^{-i p_{r}^{+} X^{-}-i p_{r}^{-} X^{+}}\left(Z_{r} \cdot \bar{Z}_{r}\right) .
$$

Finally we examine if the energy-momentum tensor $T^{X^{ \pm}}(z)$ satisfies the Virasoro algebra. In order to do so, we first derive the OPE of $\partial X^{-}(z) \partial X^{-}\left(z^{\prime}\right)$. From the correlation function

$$
\begin{aligned}
\left\langle\partial X^{-}(z) \partial X^{-}\left(Z_{0}\right)\right\rangle_{\rho}^{\mathrm{c}}= & \frac{d-26}{24}(2 i)^{2} \partial_{z} \partial_{Z_{0}} \partial_{\alpha_{0}}\left[\sum_{r=1}^{N} B_{r}^{\prime}(z)+\sum_{I=1}^{2 h-2+N} B_{I}^{\prime}(z)\right]_{\alpha_{0}=0} \quad(\mathrm{C} .24) \\
& \sim \frac{d-26}{24}(2 i)^{2} \partial_{z} \partial_{Z_{0}}\left[-\frac{2}{\left(\partial \rho\left(Z_{0}\right)\right)^{2}} \frac{1}{\left(z-Z_{0}\right)^{2}}-\frac{2 \partial^{3} \rho}{(\partial \rho)^{3}}\left(Z_{0}\right) \frac{1}{z-Z_{0}}\right],
\end{aligned}
$$

we get the OPE

$$
\begin{aligned}
\partial X^{-}(z) \partial X^{-}\left(z^{\prime}\right) \sim & -\frac{d-26}{12} \partial_{z} \partial_{z^{\prime}}\left[\frac{1}{\left(z-z^{\prime}\right)^{2}} \frac{1}{\partial X^{+}(z) \partial X^{+}\left(z^{\prime}\right)}\right] \\
\sim-\frac{d-26}{12} & {\left[-\frac{1}{\left(z-z^{\prime}\right)^{4}} \frac{6}{\left(\partial X^{+}\left(z^{\prime}\right)\right)^{2}}-\frac{1}{\left(z-z^{\prime}\right)^{3}} 3 \partial_{z^{\prime}} \frac{1}{\left(\partial X^{+}\left(z^{\prime}\right)\right)^{2}}\right.} \\
& \left.-\frac{1}{\left(z-z^{\prime}\right)^{2}} \frac{1}{2} \partial_{z^{\prime}}^{2} \frac{1}{\left(\partial X^{+}\left(z^{\prime}\right)\right)^{2}}\right] .
\end{aligned}
$$


With

$$
\begin{aligned}
\partial X^{+}(z) \partial X^{-}\left(z^{\prime}\right) & \sim \frac{1}{\left(z-z^{\prime}\right)^{2}}, \\
\partial X^{+}(z) \partial X^{+}\left(z^{\prime}\right) & \sim \text { regular }
\end{aligned}
$$

one can deduce the OPE

$$
T^{X^{ \pm}}(z) T^{X^{ \pm}}\left(z^{\prime}\right) \sim \frac{\frac{28-d}{2}}{\left(z-z^{\prime}\right)^{4}}+\frac{1}{\left(z-z^{\prime}\right)^{2}} 2 T^{ \pm}\left(z^{\prime}\right)+\frac{1}{z-z^{\prime}} \partial T^{X^{ \pm}}\left(z^{\prime}\right),
$$

which coincides with the Virasoro algebra with central charge $28-d$ as desired.

\section{Interaction points and the odd moduli}

In this appendix, we present the basic facts about the interaction points and the odd moduli in the light-cone supersheet formalism [22-24, 32].

For the supersheet coordinate

$$
\rho(\mathbf{z})=\rho_{b}(z)+\theta f(z)
$$

we define $\tilde{\mathbf{z}}_{I}$ such that $\partial \rho\left(\tilde{\mathbf{z}}_{I}\right)=\partial D \rho\left(\tilde{\mathbf{z}}_{I}\right)=0$. Since

$$
\begin{aligned}
\partial \rho & =\partial \rho_{b}+\theta \partial f, \\
\partial D \rho & =\partial f+\theta \partial^{2} \rho_{b},
\end{aligned}
$$

we find $\tilde{\mathbf{z}}_{I}=\left(\tilde{z}_{I}, \tilde{\theta}_{I}\right)$ to be

$$
\tilde{z}_{I}=z_{I}^{(b)}, \quad \tilde{\theta}_{I}=-\frac{\partial f}{\partial^{2} \rho_{b}}\left(z_{I}^{(b)}\right)
$$

where $z_{I}^{(b)}$ is one of the interaction points for $\rho_{b}(z)$, i.e. $\partial \rho_{b}\left(z_{I}^{(b)}\right)=0$. The $\rho$ coordinate corresponding to $\tilde{\mathbf{z}}_{I}$ is given as

$$
\rho\left(\tilde{\mathbf{z}}_{I}\right)=\rho_{b}\left(z_{I}^{(b)}\right)-\frac{\partial f f}{\partial^{2} \rho_{b}}\left(z_{I}^{(b)}\right) .
$$

The interaction point $\mathbf{z}_{I}=\left(z_{I}, \theta_{I}\right)$ which is superconformal covariant is the one such that for some Grassmann odd $\tilde{\xi}_{I}$

$$
\hat{\rho}(\mathbf{z})=\rho(\mathbf{z})-\rho\left(\tilde{\mathbf{z}}_{I}\right)-\left(\rho(\mathbf{z})-\rho\left(\tilde{\mathbf{z}}_{I}\right)\right)^{-\frac{1}{4}} \xi \tilde{\xi}_{I},
$$

can be expanded as

$$
\hat{\rho}(\mathbf{z})=\frac{1}{2} \partial^{2} \hat{\rho}\left(\mathbf{z}_{I}\right)\left(\mathbf{z}-\mathbf{z}_{I}\right)^{2}+\cdots,
$$

around $\mathbf{z} \sim \mathbf{z}_{I}$. Here $\xi=(\partial \rho)^{-\frac{1}{2}} D \rho$. Expanding $\rho(\mathbf{z})$ as

$$
\rho(\mathbf{z})=\rho\left(\tilde{\mathbf{z}}_{I}\right)+\tilde{\theta} \alpha+\frac{1}{2} c^{2} \tilde{z}^{2}+\tilde{z}^{2} \tilde{\theta} \beta+\tilde{z}^{2} a+\cdots,
$$


where

$$
\tilde{z}=\mathbf{z}-\tilde{\mathbf{z}}_{I}, \quad \tilde{\theta}=\theta-\tilde{\theta}_{I},
$$

we find

$$
\begin{aligned}
& \mathbf{z}_{I}-\tilde{\mathbf{z}}_{I}=-\frac{1}{c^{4}} \alpha \beta, \\
& \theta_{I}-\tilde{\theta}_{I}=\frac{a}{c^{4}} \alpha,
\end{aligned}
$$

and

$$
\begin{aligned}
\tilde{\xi}_{I} & =2^{-\frac{1}{4}} c^{-\frac{1}{2}} \alpha \\
& =2^{-\frac{1}{4}}\left(\partial^{2} \rho_{b}\right)^{-\frac{1}{4}}\left(f+\frac{f \partial f \partial^{2} f}{4\left(\partial^{2} \rho_{b}\right)^{2}}\right)\left(z_{I}^{(b)}\right) .
\end{aligned}
$$

$\tilde{\xi}_{I}$ 's are proportional to the odd supermoduli parameters in the light-cone gauge parametrization. For our purpose, it is convenient to define

$$
\xi_{I} \equiv 2\left(\partial^{2} \rho_{b}\right)^{-\frac{1}{4}}\left(f+\frac{f \partial f \partial^{2} f}{4\left(\partial^{2} \rho_{b}\right)^{2}}\right)\left(z_{I}^{(b)}\right) .
$$

From (D.9), we get

$$
\rho\left(\mathbf{z}_{I}\right)=\rho\left(\tilde{\mathbf{z}}_{I}\right)+\left(\theta_{I}-\tilde{\theta}_{I}\right) D \rho\left(\tilde{\mathbf{z}}_{I}\right),
$$

but since $\alpha=D \rho\left(\tilde{\mathbf{z}}_{I}\right)$, we find

$$
\rho\left(\mathbf{z}_{I}\right)=\rho\left(\tilde{\mathbf{z}}_{I}\right)
$$

\section{E Calculations of correlation functions of supersymmetric $X^{ \pm}$CFT}

In this appendix, we present some details of the calculation of the correlation functions of supersymmetric $X^{ \pm}$CFT.

$\left\langle D \mathcal{X}^{-}(\mathbf{z})\right\rangle_{\rho}$ in $(3.61)$ can be expressed as

$$
\begin{aligned}
\left\langle D \mathcal{X}^{-}(\mathbf{z})\right\rangle_{\rho}= & \frac{d-10}{8} 2 i D\left[\frac{1}{2}\left(\sum_{r} B_{r}(z, \bar{z})+\sum_{I} B_{I}(z, \bar{z})\right)\right. \\
& \left.+N(\mathbf{z}, \overline{\mathbf{z}})+\sum_{r} N_{r}(\mathbf{z}, \overline{\mathbf{z}})+\sum_{I} N_{I}(\mathbf{z}, \overline{\mathbf{z}})\right]
\end{aligned}
$$

where

$$
\begin{aligned}
& \sum_{r} B_{r}\left(Z_{0}, \bar{Z}_{0}\right)+\sum_{I} B_{I}\left(Z_{0}, \bar{Z}_{0}\right)-\sum_{r} B_{r}\left(Z_{N+1}, \bar{Z}_{N+1}\right)-\sum_{I} B_{I}\left(Z_{N+1}, \bar{Z}_{N+1}\right) \\
&=\left.\partial_{\alpha_{0}}\left(-\Gamma^{\prime}\right)\right|_{\alpha_{0}=0}, \\
& N\left(\mathbf{Z}_{0}, \overline{\mathbf{Z}}_{0}\right)=\left.\partial_{\alpha_{0}}\left(-\delta \Gamma_{0}^{\prime}-\delta \Gamma_{I^{(0)}}^{\prime}\right)\right|_{\alpha_{0}=0} \\
& N_{r}\left(\mathbf{Z}_{0}, \overline{\mathbf{Z}}_{0}\right)-N_{r}\left(\mathbf{Z}_{N+1}, \overline{\mathbf{Z}}_{N+1}\right)=\left.\partial_{\alpha_{0}}\left(-\delta \Gamma_{r}^{\prime}\right)\right|_{\alpha_{0}=0} \\
& N_{I}\left(\mathbf{Z}_{0}, \overline{\mathbf{Z}}_{0}\right)-N_{I}\left(\mathbf{Z}_{N+1}, \overline{\mathbf{Z}}_{N+1}\right)=\left.\partial_{\alpha_{0}}\left(-\delta \Gamma_{I}^{\prime}\right)\right|_{\alpha_{0}=0}
\end{aligned}
$$


It is straightforward to calculate these terms. $B_{r}(z, \bar{z})$ and $B_{I}(z, \bar{z})$ essentially coincide with the bosonic ones

$$
\begin{aligned}
& B_{r}(z, \bar{z})=\frac{1}{\alpha_{r}}\left(g\left(Z_{r}, z\right)-g\left(z_{I^{(r)}}, z\right)\right)+\text { c.c. } \\
& B_{I}(z, \bar{z})=\frac{\partial^{3} \rho_{b}}{2\left(\partial^{2} \rho_{b}\right)^{2}}\left(z_{I}\right) \frac{\partial}{\partial z_{I}} g\left(z_{I}, z\right)-\frac{3}{2 \partial^{2} \rho_{b}\left(z_{I}\right)} \frac{\partial^{2}}{\partial z_{I}^{2}} g\left(z_{I}, z\right)+\text { c.c. } \\
& g(z, w)=\ln E(z, w)-2 \pi i \int_{P_{0}}^{z} \omega \frac{1}{\operatorname{Im} \Omega} \operatorname{Im} \int_{P_{0}}^{w} \omega .
\end{aligned}
$$

In order to evaluate $N(\mathbf{z}, \overline{\mathbf{z}})$, we need $z_{I^{(0)}}^{(b)}-Z_{0}$ up to $\mathcal{O}\left(\alpha_{0}^{2}\right)$, which is given by the second equation in (C.3). Using this, it is straightforward to get

$$
\begin{aligned}
N(\mathbf{z}, \overline{\mathbf{z}})= & -\frac{2 \partial^{2} f f}{\left(\partial \rho_{b}\right)^{3}}(z)+\frac{4 \partial^{2} \rho_{b} \partial f f}{\left(\partial \rho_{b}\right)^{4}}(z) \\
& +\theta\left(-\frac{\partial^{2} f \partial f f}{2\left(\partial \rho_{b}\right)^{4}}+\frac{2 \partial^{2} f}{\left(\partial \rho_{b}\right)^{2}}-\frac{4 \partial^{2} \rho_{b} \partial f}{\left(\partial \rho_{b}\right)^{3}}+\frac{5\left(\partial^{2} \rho_{b}\right)^{2} f}{2\left(\partial \rho_{b}\right)^{4}}-\frac{\partial^{3} \rho_{b} f}{\left(\partial \rho_{b}\right)^{3}}\right)(z) \\
& + \text { c.c. }
\end{aligned}
$$

This expression does not involve $Z_{N+1}$ or $\Theta_{N+1}$. In the same way, one can show that $\left.\partial_{\alpha_{0}}\left(-\delta \Gamma_{N+1}^{\prime}-\delta \Gamma_{I^{(N+1)}}^{\prime}\right)\right|_{\alpha_{0}=0}$ does not involve $Z_{0}$ or $\Theta_{0}$ and we do not have to include the contributions from this term in (E.1). $N_{r}(\mathbf{z}, \overline{\mathbf{z}})$ and $N_{I}(\mathbf{z}, \overline{\mathbf{z}})$ can be evaluated by simply calculating the right hand sides of in (E.2). The results are in the form on the left hand sides of these equations and we obtain

$$
\begin{aligned}
& N_{r}(\mathbf{z}, \overline{\mathbf{z}}) \\
& =\frac{1}{2 \alpha_{r}}\left[\left\{-\frac{\partial^{2} f f}{\left(\partial^{2} \rho_{b}\right)^{2}}+\frac{\partial^{3} \rho_{b} \partial f f}{\left(\partial^{2} \rho_{b}\right)^{3}}\right\}\left(z_{I^{(r)}}^{(b)}\right) \partial_{z_{I^{(r)}}^{(b)}} g\left(z_{I^{(r)}}^{(b)}, z\right)-\frac{\partial f f}{\left(\partial^{2} \rho_{b}\right)^{2}} \partial_{z_{I^{(r)}}^{(b)}}^{2} g\left(z_{I^{(r)}}^{(b)}, z\right)\right. \\
& \left.+\theta\left\{\frac{\partial f}{\partial^{2} \rho_{b}}\left(z_{I^{(r)}}^{(b)}\right) S_{\delta}\left(z_{I^{(r)}}^{(b)}, z\right)-\frac{f}{\partial^{2} \rho_{b}}\left(z_{I^{(r)}}^{(b)}\right) \partial_{z_{I^{(r)}}^{(b)}} S_{\delta}\left(z_{I^{(r)}}^{(b)}, z\right)\right\}\right] \\
& + \text { c.c. } \\
& N_{I}(\mathbf{z}, \overline{\mathbf{z}}) \\
& =\left\{\frac{\partial^{4} f \partial^{2} f \partial f f}{12\left(\partial^{2} \rho_{b}\right)^{5}}-\frac{2\left(\partial^{4} f f+\partial^{3} f \partial f\right)}{3\left(\partial^{2} \rho_{b}\right)^{3}}-\frac{\partial^{3} \rho_{b} \partial^{3} f \partial^{2} f \partial f f}{3\left(\partial^{2} \rho_{b}\right)^{6}}\right. \\
& +\frac{\partial^{3} \rho_{b}\left(\frac{7}{3} \partial^{3} f f+\partial^{2} f \partial f\right)}{\left(\partial^{2} \rho_{b}\right)^{4}}-\frac{15\left(\partial^{3} \rho_{b}\right)^{2} \partial^{2} f f}{4\left(\partial^{2} \rho_{b}\right)^{5}} \\
& \left.+\frac{17 \partial^{4} \rho_{b} \partial^{2} f f}{12\left(\partial^{2} \rho_{b}\right)^{4}}+\left(\frac{3\left(\partial^{3} \rho_{b}\right)^{3}}{\left(\partial^{2} \rho_{b}\right)^{6}}-\frac{11 \partial^{4} \rho_{b} \partial^{3} \rho_{b}}{4\left(\partial^{2} \rho_{b}\right)^{5}}+\frac{5 \partial^{5} \rho_{b}}{12\left(\partial^{2} \rho_{b}\right)^{4}}\right) \partial f f\right\}\left(z_{I}^{(b)}\right) \partial_{z_{I}^{(b)}} g\left(z_{I}^{(b)}, z\right) \\
& +\left\{\frac{\partial^{3} f \partial^{2} f \partial f f}{3\left(\partial^{2} \rho_{b}\right)^{5}}-\frac{4 \partial^{3} f f}{3\left(\partial^{2} \rho_{b}\right)^{3}}\right. \\
& \left.+\frac{3 \partial^{3} \rho_{b} \partial^{2} f f}{\left(\partial^{2} \rho_{b}\right)^{4}}-\frac{3\left(\partial^{3} \rho_{b}\right)^{2} \partial f f}{\left(\partial^{2} \rho_{b}\right)^{5}}+\frac{5 \partial^{4} \rho_{b} \partial f f}{4\left(\partial^{2} \rho_{b}\right)^{4}}\right\}\left(z_{I}^{(b)}\right) \partial_{z_{I}^{(b)}}^{2} g\left(z_{I}^{(b)}, z\right) \\
& +\left\{-\frac{\partial^{2} f f}{\left(\partial^{2} \rho_{b}\right)^{3}}+\frac{3 \partial^{3} \rho_{b} \partial f f}{2\left(\partial^{2} \rho_{b}\right)^{4}}\right\}\left(z_{I}^{(b)}\right) \partial_{z_{I}^{(b)}}^{3} g\left(z_{I}^{(b)}, z\right)-\frac{5 \partial f f}{12\left(\partial^{2} \rho_{b}\right)^{3}}\left(z_{I}^{(b)}\right) \partial_{z_{I}^{(b)}}^{4} g\left(z_{I}^{(b)}, z\right)
\end{aligned}
$$




$$
\begin{aligned}
+\theta[ & \left\{-\frac{\partial^{3} f \partial^{2} f \partial f}{12\left(\partial^{2} \rho_{b}\right)^{4}}+\frac{2 \partial^{3} f}{3\left(\partial^{2} \rho_{b}\right)^{2}}-\frac{\partial^{3} \rho_{b} \partial^{2} f}{\left(\partial^{2} \rho_{b}\right)^{3}}+\frac{3\left(\partial^{3} \rho_{b}\right)^{2} \partial f}{4\left(\partial^{2} \rho_{b}\right)^{4}}-\frac{5 \partial^{4} \rho_{b} \partial f}{12\left(\partial^{2} \rho_{b}\right)^{3}}\right\}\left(z_{I}^{(b)}\right) S_{\delta}\left(z_{I}^{(b)}, z\right) \\
& +\left\{\frac{\partial^{3} f \partial^{2} f f}{12\left(\partial^{2} \rho_{b}\right)^{4}}-\frac{3\left(\partial^{3} \rho_{b}\right)^{2} f}{4\left(\partial^{2} \rho_{b}\right)^{4}}+\frac{5 \partial^{4} \rho_{b} f}{12\left(\partial^{2} \rho_{b}\right)^{3}}\right\}\left(z_{I}^{(b)}\right) \partial_{z_{I}^{(b)}} S_{\delta}\left(z_{I}^{(b)}, z\right) \\
& +\left\{-\frac{\partial^{3} f \partial f f}{12\left(\partial^{2} \rho_{b}\right)^{4}}+\frac{\partial^{3} \rho_{b} f}{\left(\partial^{2} \rho_{b}\right)^{3}}\right\}\left(z_{I}^{(b)}\right) \partial_{z_{I}^{(b)}}^{2} S_{\delta}\left(z_{I}^{(b)}, z\right) \\
& \left.+\left\{\frac{\partial^{2} f \partial f f}{12\left(\partial^{2} \rho_{b}\right)^{4}}-\frac{2 f}{3\left(\partial^{2} \rho_{b}\right)^{2}}\right\}\left(z_{I}^{(b)}\right) \partial_{z_{I}^{(b)}}^{3} S_{\delta}\left(z_{I}^{(b)}, z\right)\right] \\
& + \text { c.c. }
\end{aligned}
$$

A few comments are in order:

- $\left\langle D \mathcal{X}^{-}(\mathbf{z})\right\rangle_{\rho}$ given in (E.1) is meromorphic with respect to $\mathbf{z}$.

- $\mathcal{X}^{ \pm}$involves auxiliary field $F^{ \pm}$as its component. With the action (3.1), the equation of motion implies $F^{+}=0 . F^{+}$can be nonzero at $z=Z_{r}$ where $\mathcal{X}^{-}$is inserted. (E.1) implies that the expectation value of $F^{-}$vanishes even with the sources.

\section{E.1 An expression in terms of the superfield $\rho(\mathrm{z})$}

It is possible to rewrite $\left\langle D \mathcal{X}^{-}(\mathbf{z})\right\rangle_{\rho}$ given in (E.1) in terms of the superfield $\rho(\mathbf{z})$, the covariant derivative and the fermionic coordinate $\theta$ as

$$
\left\langle D \mathcal{X}^{-}(\mathbf{z})\right\rangle_{\rho}=\frac{d-10}{8} 2 i D \operatorname{Re}\left[\sum_{r}\left(\frac{1}{\alpha_{r}} g\left(Z_{r}, z\right)+\mathcal{K}_{1}\left(\tilde{\mathbf{z}}_{I^{(r)}} ; \mathbf{z}\right)\right)+\mathcal{K}_{2}(\mathbf{z})+\sum_{I} \mathcal{K}_{3}\left(\tilde{\mathbf{z}}_{I} ; \mathbf{z}\right)\right]
$$

where

$$
\begin{aligned}
& \mathcal{K}_{1}\left(\tilde{\mathbf{z}}_{I^{(r)}} ; \mathbf{z}\right) \\
& =\frac{1}{\alpha_{r}}\left[-g\left(\tilde{\mathbf{z}}_{I^{(r)}}, \mathbf{z}\right)-\partial g\left(\tilde{\mathbf{z}}_{I^{(r)}}, \mathbf{z}\right) \frac{\partial^{2} D \rho D \rho}{\left(\partial^{2} \rho\right)}\left(\tilde{\mathbf{z}}_{I^{(r)}}\right)+\partial D\left(g\left(\tilde{\mathbf{z}}_{I^{(r)}}, \mathbf{z}\right)\right) \frac{D \rho}{\partial^{2} \rho}\left(\tilde{\mathbf{z}}_{I^{(r)}}\right)\right], \\
& \mathcal{K}_{2}(\mathbf{z})=\left(8 \frac{\partial^{2} \rho \partial D \rho D \rho}{(\partial \rho)^{4}}-4 \frac{\partial^{2} D \rho D \rho}{(\partial \rho)^{3}}\right)(\mathbf{z}) \\
& \quad+\theta\left(-3 \frac{\left(\partial^{2} \rho\right)^{2}}{(\partial \rho)^{4}}+2 \frac{\partial^{3} \rho}{(\partial \rho)^{3}}+3 \frac{\partial^{2} D \rho \partial D \rho}{(\partial \rho)^{4}}\right) D \rho(\mathbf{z}),
\end{aligned}
$$

$\mathcal{K}_{3}\left(\mathbf{z}_{I} ; \mathbf{z}\right)$

$$
\begin{aligned}
= & D\left(g\left(\tilde{\mathbf{z}}_{I}, \mathbf{z}\right)\right)\left(2 \frac{\partial^{3} \rho \partial^{2} D \rho}{\left(\partial^{2} \rho\right)^{3}}-\frac{4}{3} \frac{\partial^{3} D \rho}{\left(\partial^{2} \rho\right)^{2}}\right)\left(\tilde{\mathbf{z}}_{I}\right) \\
+ & \partial\left(g\left(\tilde{\mathbf{z}}_{I}, \mathbf{z}\right)\right)\left\{\frac{14}{3} \frac{\partial^{3} D \rho \partial^{3} \rho D \rho}{\left(\partial^{2} \rho\right)^{4}}-\frac{4}{3} \frac{\partial^{4} D \rho D \rho}{\left(\partial^{2} \rho\right)^{3}}\right. \\
& \left.+\left(-\frac{15}{2} \frac{\left(\partial^{3} \rho\right)^{2}}{\left(\partial^{2} \rho\right)^{5}}+\frac{17}{6} \frac{\partial^{4} \rho}{\left(\partial^{2} \rho\right)^{4}}\right) \partial^{2} D \rho D \rho+\frac{1}{2} \frac{\partial^{3} \rho}{\left(\partial^{2} \rho\right)^{2}}\right\}\left(\tilde{\mathbf{z}}_{I}\right) \\
& +\partial D\left(g\left(\tilde{\mathbf{z}}_{I}, \mathbf{z}\right)\right)\left(-\frac{1}{6} \frac{\partial^{3} D \rho \partial^{2} D \rho D \rho}{\left(\partial^{2} \rho\right)^{4}}+\frac{3}{2} \frac{\left(\partial^{3} \rho\right)^{2} D \rho}{\left(\partial^{2} \rho\right)^{4}}-\frac{5}{6} \frac{\partial^{4} \rho D \rho}{\left(\partial^{2} \rho\right)^{3}}\right)\left(\tilde{\mathbf{z}}_{I}\right) \\
& +\partial^{2}\left(g\left(\tilde{\mathbf{z}}_{I}, \mathbf{z}\right)\right)\left(6 \frac{\partial^{3} \rho \partial^{2} D \rho D \rho}{\left(\partial^{2} \rho\right)^{4}}-\frac{8}{3} \frac{\partial^{3} D \rho D \rho}{\left(\partial^{2} \rho\right)^{3}}-\frac{3}{2} \frac{1}{\partial^{2} \rho}\right)\left(\tilde{\mathbf{z}}_{I}\right)
\end{aligned}
$$




$$
\begin{aligned}
& -2 \partial^{2} D\left(g\left(\tilde{\mathbf{z}}_{I}, \mathbf{z}\right)\right) \frac{\partial^{3} \rho D \rho}{\left(\partial^{2} \rho\right)^{3}}\left(\tilde{\mathbf{z}}_{I}\right)-2 \partial^{3}\left(g\left(\tilde{\mathbf{z}}_{I}, \mathbf{z}\right)\right) \frac{\partial^{2} D \rho D \rho}{\left(\partial^{2} \rho\right)^{3}}\left(\tilde{\mathbf{z}}_{I}\right) \\
& +\frac{4}{3} \partial^{3} D\left(g\left(\tilde{\mathbf{z}}_{I}, \mathbf{z}\right)\right) \frac{D \rho}{\left(\partial^{2} \rho\right)^{2}}\left(\tilde{\mathbf{z}}_{I}\right) \\
& +\tilde{\theta}_{I}\left[D\left(g\left(\tilde{\mathbf{z}}_{I}, \mathbf{z}\right)\right)\left(\frac{1}{2} \frac{\left(\partial^{3} \rho\right)^{2}}{\left(\partial^{2} \rho\right)^{3}}-\frac{1}{2} \frac{\partial^{4} \rho}{\left(\partial^{2} \rho\right)^{2}}-\frac{1}{2} \frac{\partial^{3} D \rho \partial^{2} D \rho}{\left(\partial^{2} \rho\right)^{3}}\right)\left(\tilde{\mathbf{z}}_{I}\right)\right. \\
& +\partial\left(g\left(\tilde{\mathbf{z}}_{I}, \mathbf{z}\right)\right)\left\{-3 \frac{\partial^{3} \rho \partial^{3} D \rho \partial^{2} D \rho D \rho}{\left(\partial^{2} \rho\right)^{5}}+\frac{\partial^{4} D \rho \partial^{2} D \rho D \rho}{\left(\partial^{2} \rho\right)^{4}}\right. \\
& +\left(\frac{3}{2} \frac{\left(\partial^{3} \rho\right)^{3}}{\left(\partial^{2} \rho\right)^{5}}-2 \frac{\partial^{4} \rho \partial^{3} \rho}{\left(\partial^{2} \rho\right)^{4}}+\frac{1}{2} \frac{\partial^{5} \rho}{\left(\partial^{2} \rho\right)^{3}}\right) D \rho \\
& +\frac{\partial^{3} \rho \partial^{2} D \rho}{\left.\left(\partial^{2} \rho\right)^{3}-\frac{1}{2} \frac{\partial^{3} D \rho}{\left(\partial^{2} \rho\right)^{2}}\right\}\left(\tilde{\mathbf{z}}_{I}\right)} \\
& +\partial D\left(g\left(\tilde{\mathbf{z}}_{I}, \mathbf{z}\right)\right)\left\{-\frac{3}{2} \frac{\partial^{3} \rho \partial^{3} D \rho D \rho}{\left(\partial^{2} \rho\right)^{4}}+\frac{1}{2} \frac{\partial^{4} D \rho D \rho}{\left(\partial^{2} \rho\right)^{3}}\right. \\
& \left.+\left(\frac{3}{2} \frac{\left(\partial^{3} \rho\right)^{2}}{\left(\partial^{2} \rho\right)^{5}}-\frac{1}{2} \frac{\partial^{4} \rho}{\left(\partial^{2} \rho\right)^{4}}\right) \partial^{2} D \rho D \rho-\frac{1}{2} \frac{\partial^{3} \rho}{\left(\partial^{2} \rho\right)^{2}}\right\}\left(\tilde{\mathbf{z}}_{I}\right) \\
& +\partial^{2}\left(g\left(\tilde{\mathbf{z}}_{I}, \mathbf{z}\right)\right)\left\{\left(-\frac{3}{2} \frac{\left(\partial^{3} \rho\right)^{2}}{\left(\partial^{2} \rho\right)^{4}}+\frac{\partial^{4} \rho}{\left(\partial^{2} \rho\right)^{3}}\right) D \rho+\frac{3}{2} \frac{\partial^{3} D \rho \partial^{2} D \rho D \rho}{\left(\partial^{2} \rho\right)^{4}}-\frac{3}{2} \frac{\partial^{2} D \rho}{\left(\partial^{2} \rho\right)^{2}}\right\}\left(\tilde{\mathbf{z}}_{I}\right) \\
& +\partial^{2} D\left(g\left(\tilde{\mathbf{z}}_{I}, \mathbf{z}\right)\right)\left(\frac{1}{2} \frac{\partial^{3} D \rho D \rho}{\left(\partial^{2} \rho\right)^{3}}+\frac{3}{2} \frac{1}{\partial^{2} \rho}\right)\left(\tilde{\mathbf{z}}_{I}\right) \\
& +\partial^{3}\left(g\left(\tilde{\mathbf{z}}_{I}, \mathbf{z}\right)\right) \frac{\partial^{3} \rho D \rho}{\left(\partial^{2} \rho\right)^{3}}\left(\tilde{\mathbf{z}}_{I}\right)-\frac{1}{2} \partial^{3} D\left(g\left(\tilde{\mathbf{z}}_{I}, \mathbf{z}\right)\right) \frac{\partial^{2} D \rho D \rho}{\left(\partial^{2} \rho\right)^{3}}\left(\tilde{\mathbf{z}}_{I}\right) \\
& \left.-\frac{1}{2} \partial^{4}\left(g\left(\tilde{\mathbf{z}}_{I}, \mathbf{z}\right)\right) \frac{D \rho}{\left(\partial^{2} \rho\right)^{2}}\left(\tilde{\mathbf{z}}_{I}\right)\right], \\
& +
\end{aligned}
$$

where the derivatives act on the first arguments of $g\left(\tilde{\mathbf{z}}_{I^{(r)}}, \mathbf{z}\right)$ and $g\left(\tilde{\mathbf{z}}_{I}, \mathbf{z}\right)$. This expression shall be useful in doing calculations.

\section{E.2 Energy-momentum tensor}

Now we can discuss the properties of the energy-momentum tensor superfield $T^{\mathcal{X}^{ \pm}}(\mathbf{z})$ given in (3.62). For example, the correlation functions with one $T^{\mathcal{X}^{ \pm}}(\mathbf{z})$ insertion can be given by

$$
\begin{aligned}
& \left\langle T^{\mathcal{X}^{ \pm}}(\mathbf{z}) \prod_{r=1}^{N} e^{-i p_{r}^{+} \mathcal{X}^{-}}\left(\mathbf{Z}_{r}, \overline{\mathbf{Z}}_{r}\right) \prod_{s=1}^{M} e^{-i p_{s}^{-} \mathcal{X}^{+}}\left(\mathbf{w}_{s}, \overline{\mathbf{w}}_{s}\right)\right\rangle_{\hat{g}_{z \bar{z}}}^{\mathcal{X}^{ \pm}}
\end{aligned}
$$

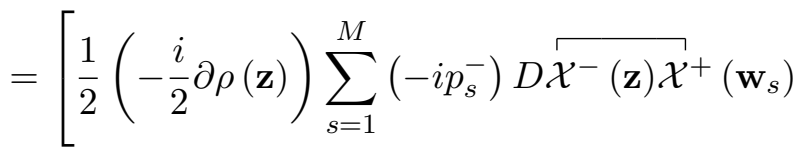

$$
\begin{aligned}
& \left.+\frac{1}{2}\left(-\frac{i}{2} D \rho(\mathbf{z})\right) \sum_{s=1}^{M}\left(-i p_{s}^{-}\right) \partial \widetilde{\mathcal{X}^{-}(\mathbf{z}) \mathcal{X}^{+}}\left(\mathbf{w}_{s}\right)+\left\langle T^{\mathcal{X}^{ \pm}}(\mathbf{z})\right\rangle_{\rho}\right] \\
& \times\left\langle\prod_{r=1}^{N} e^{-i p_{r}^{+} \mathcal{X}^{-}}\left(\mathbf{Z}_{r}, \overline{\mathbf{Z}}_{r}\right) \prod_{s=1}^{M} e^{-i p_{s}^{-} \mathcal{X}^{+}}\left(\mathbf{w}_{s}, \overline{\mathbf{w}}_{s}\right)\right\rangle_{\hat{g}_{z \bar{z}}}^{X^{ \pm}},
\end{aligned}
$$


where

$$
\begin{aligned}
\left\langle T^{\mathcal{X}^{ \pm}}(\mathbf{z})\right\rangle_{\rho}= & \frac{1}{2}\left(-\frac{i}{2} \partial \rho(\mathbf{z})\left\langle D \mathcal{X}^{-}(\mathbf{z})\right\rangle_{\rho}-\frac{i}{2} D \rho(\mathbf{z})\left\langle\partial \mathcal{X}^{-}(\mathbf{z})\right\rangle_{\rho}\right)-\frac{d-10}{4} S(\mathbf{z}, \boldsymbol{\rho}) \\
& +\frac{1}{2} \lim _{\mathbf{w} \rightarrow \mathbf{z}}\left(\partial \widehat{\mathcal{X}^{+}(\mathbf{w}) D \mathcal{X}^{-}}(\mathbf{z})-\partial_{\mathbf{w}} D_{\mathbf{z}} \ln (\mathbf{w}-\mathbf{z})\right) \\
& +\frac{1}{2} \lim _{\mathbf{w} \rightarrow \mathbf{z}}\left(D \mathcal{X}^{+}(\mathbf{w}) \partial \mathcal{X}^{-}(\mathbf{z})-D_{\mathbf{w}} \partial_{\mathbf{z}} \ln (\mathbf{w}-\mathbf{z})\right)
\end{aligned}
$$

It is straightforward to calculate correlation functions of $T^{\mathcal{X}^{ \pm}}(\mathbf{z})$ and show the properties of the supersymmetric $X^{ \pm}$CFT mentioned at the end of subsection 3.3, using the expression (E.1) or (E.6). Since these calculations are not so illuminating, we do not reproduce them here.

Open Access. This article is distributed under the terms of the Creative Commons Attribution License (CC-BY 4.0), which permits any use, distribution and reproduction in any medium, provided the original author(s) and source are credited.

\section{References}

[1] Y. Baba, N. Ishibashi and K. Murakami, Light-Cone Gauge String Field Theory in Noncritical Dimensions, JHEP 12 (2009) 010 [arXiv:0909.4675] [INSPIRE].

[2] Y. Baba, N. Ishibashi and K. Murakami, Light-cone Gauge NSR Strings in Noncritical Dimensions, JHEP 01 (2010) 119 [arXiv:0911.3704] [INSPIRE].

[3] Y. Baba, N. Ishibashi and K. Murakami, Light-Cone Gauge Superstring Field Theory and Dimensional Regularization, JHEP 10 (2009) 035 [arXiv:0906.3577] [INSPIRE].

[4] Y. Baba, N. Ishibashi and K. Murakami, Light-cone Gauge Superstring Field Theory and Dimensional Regularization II, JHEP 08 (2010) 102 [arXiv:0912.4811] [INSPIRE].

[5] N. Ishibashi and K. Murakami, Light-cone Gauge NSR Strings in Noncritical Dimensions II - Ramond Sector, JHEP 01 (2011) 008 [arXiv:1011.0112] [INSPIRE].

[6] N. Ishibashi and K. Murakami, Spacetime Fermions in Light-cone Gauge Superstring Field Theory and Dimensional Regularization, JHEP 07 (2011) 090 [arXiv:1103.2220] [INSPIRE].

[7] J. Greensite and F.R. Klinkhamer, New Interactions for Superstrings, Nucl. Phys. B 281 (1987) 269 [INSPIRE].

[8] J. Greensite and F.R. Klinkhamer, Superstring Amplitudes and Contact Interactions, Nucl. Phys. B 304 (1988) 108 [inSPIRE].

[9] J. Greensite and F.R. Klinkhamer, Contact Interactions in Closed Superstring Field Theory, Nucl. Phys. B 291 (1987) 557 [inSPIRE].

[10] M.B. Green and N. Seiberg, Contact Interactions in Superstring Theory, Nucl. Phys. B 299 (1988) 559 [INSPIRE].

[11] C. Wendt, Scattering Amplitudes and Contact Interactions in Witten's Superstring Field Theory, Nucl. Phys. B 314 (1989) 209 [inSPIRE]. 
[12] N. Ishibashi and K. Murakami, Multiloop Amplitudes of Light-cone Gauge Bosonic String Field Theory in Noncritical Dimensions, JHEP 09 (2013) 053 [arXiv:1307.6001] [INSPIRE].

[13] E. D'Hoker and D.H. Phong, The Geometry of String Perturbation Theory, Rev. Mod. Phys. 60 (1988) 917 [INSPIRE].

[14] S. Mandelstam, The interacting string picture and functional integration, in proceedings of Workshop on unified string theories, Santa Barbara, CA, U.S.A. 29 July-16 August 1985.

[15] L. Álvarez-Gaumé, J.B. Bost, G.W. Moore, P.C. Nelson and C. Vafa, Bosonization on Higher Genus Riemann Surfaces, Commun. Math. Phys. 112 (1987) 503 [INSPIRE].

[16] E.P. Verlinde and H.L. Verlinde, Chiral Bosonization, Determinants and the String Partition Function, Nucl. Phys. B 288 (1987) 357 [InSPIRE].

[17] M.J. Dugan and H. Sonoda, Functional determinants on Riemann surfaces, Nucl. Phys. B 289 (1987) 227 [INSPIRE].

[18] H. Sonoda, Functional Determinants on Punctured Riemann Surfaces and Their Application to String Theory, Nucl. Phys. B 294 (1987) 157 [INSPIRE].

[19] R. Wentworth, The asymptotics of the arakelov-green's function and faltings' delta invariant, Commun. Math. Phys. 137 (1991) 427.

[20] R.A. Wentworth, Precise constants in bosonization formulas on Riemann surfaces. I, Commun. Math. Phys. 282 (2008) 339.

[21] G. Faltings, Calculus on arithmetic surfaces, Ann. Math. 119 (1984) 387.

[22] N. Berkovits, Calculation of Scattering Amplitudes for the Neveu-Schwarz Model Using Supersheet Functional Integration, Nucl. Phys. B 276 (1986) 650 [INSPIRE].

[23] N. Berkovits, Supersheet Functional Integration and the Interacting Neveu-Schwarz String, Nucl. Phys. B 304 (1988) 537 [inSPIRE].

[24] K. Aoki, E. D'Hoker and D.H. Phong, Unitarity of Closed Superstring Perturbation Theory, Nucl. Phys. B 342 (1990) 149 [InSPIRE].

[25] N. Berkovits, Supersheet Functional Integration and the Calculation of Nsr Scattering Amplitudes Involving Arbitrarily Many External Ramond Strings, Nucl. Phys. B 331 (1990) 659 [INSPIRE].

[26] N. Berkovits, The Heterotic Green-Schwarz superstring on an $N=(2,0)$ superworldsheet, Nucl. Phys. B 379 (1992) 96 [hep-th/9201004] [INSPIRE].

[27] N. Berkovits, Calculation of Green-Schwarz superstring amplitudes using the $N=2$ twistor string formalism, Nucl. Phys. B 395 (1993) 77 [hep-th/9208035] [INSPIRE].

[28] N.J. Berkovits and J.M. Maldacena, $N=2$ superconformal description of superstring in Ramond-Ramond plane wave backgrounds, JHEP 10 (2002) 059 [hep-th/0208092] [INSPIRE].

[29] M.B. Green and J.H. Schwarz, Superstring Interactions, Nucl. Phys. B 218 (1983) 43 [INSPIRE].

[30] M.B. Green, J.H. Schwarz and L. Brink, Superfield Theory of Type II Superstrings, Nucl. Phys. B 219 (1983) 437 [inSPIRE].

[31] E. D'Hoker and D.H. Phong, Functional determinants on Mandelstam diagrams, Commun. Math. Phys. 124 (1989) 629 [inSPIRE].

[32] S. Mandelstam, The $n$ loop string amplitude: Explicit formulas, finiteness and absence of ambiguities, Phys. Lett. B 277 (1992) 82 [INSPIRE]. 